\title{
JP-10 combustion studied with shock tube experiments and modeled with automatic reaction mechanism generation
}

Connie W. Gao, Aäron Vandeputte, Nathan W. Yee, William H. Green*

Massachusetts Institute of Technology, Department of Chemical Engineering

77 Massachusetts Avenue, Cambridge MA 02139

Robin E. Bonomi ${ }^{\dagger}$, Gregory R. Magoon, Hsi-Wu Wong", Oluwayemisi O. Oluwole, David K. Lewis ${ }^{\dagger}$

Aerodyne Research, Inc.

45 Manning Road, Billerica MA 01821-397

Nick M. Vandewiele, Kevin M. Van Geem

Universiteit Gent, Laboratory for Chemical Technology

Technologiepark 914, B-9052, Gent, Belgium

*Corresponding author

\section{TOC}

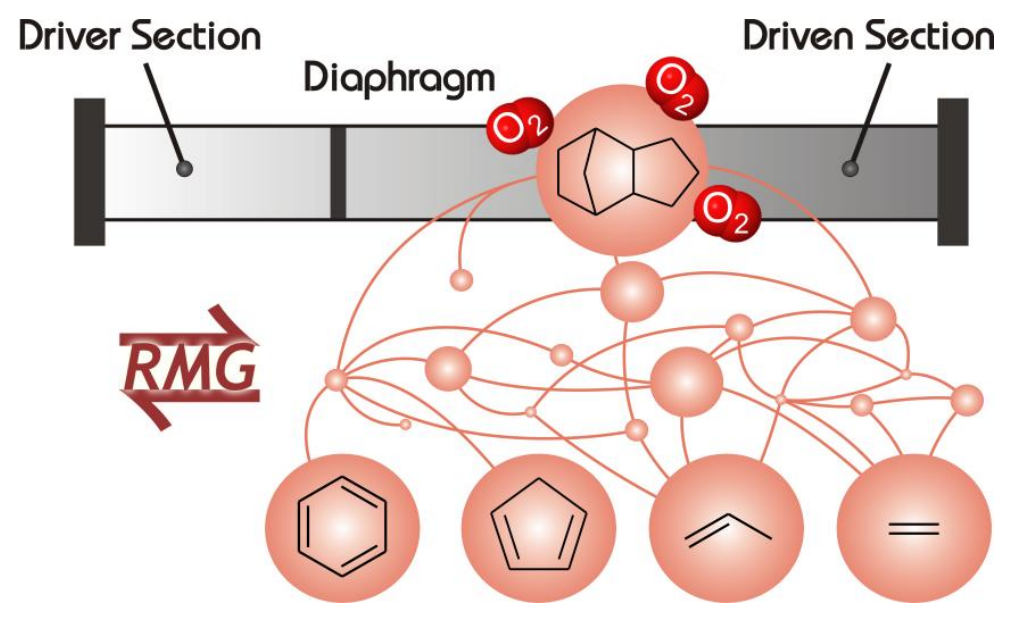

${ }^{\dagger}$ Also: Department of Chemistry, Connecticut College, New London, CT 06320

$\$$ Current address: Department of Chemical Engineering, University of Massachusetts Lowell, Lowell, MA 01854

1 


\begin{abstract}
This work presents shock tube experiments and kinetic modeling efforts on the pyrolysis and combustion of JP-10. The experiments were performed at 6-8 atm using $2000 \mathrm{ppm}$ of JP-10 over a temperature range of $1000-1600 \mathrm{~K}$ for pyrolysis and oxidation equivalence ratios from 0.14 to 1.0. This work distinguishes itself from previous studies as GC/MS was used to identify and quantify the products within the shocked samples, enabling the tracking of product yield dependence on equivalence ratio as well as identifying several new intermediates that form during JP-10's decomposition. A detailed, comprehensive model of JP-10's combustion and pyrolysis kinetics was constructed with the help of RMG, an open-source reaction mechanism generation software package. The resulting model, which includes 691 species reacting in 15518 reactions, was extensively validated against the shock tube experimental dataset as well as newly published flow tube pyrolysis data from Ghent. Most of the important rate coefficients were computed using quantum chemistry. The model succeeds in identifying all major pyrolysis and combustion products and captures key trends in the product distribution. Simulated ignition delays agree within a factor of 4 with most experimental ignition delay data gathered from literature. The presented experimental work and modeling efforts yield new insights on JP-10's complex decomposition and oxidation chemistry and identifies key pathways towards aromatics formation.
\end{abstract}

Keywords: JP-10, combustion kinetics, shock tube, automatic reaction mechanism generation

\title{
Introduction
}

JP-10 is a synthetic aviation turbine fuel that is essentially a pure component, i.e. exotetrahydrodicyclopentadiene, $\mathrm{C}_{10} \mathrm{H}_{16}$ (Figure 1). Its low freezing point, high volumetric energy density, and high specific impulse make it one of the most promising air-breathing missile fuels. ${ }^{1}$ Due to its success, JP-10 prices skyrocketed and almost doubled from 2006 to 2010, driving further research into more efficient use of this expensive fuel, such as blending JP-10 with cheaper alternatives. Major research efforts have also been focused on the development of propulsion technologies, including ramjet, scramjet and pulse detonation engines, using JP-10 as the predominant fuel. JP-10 is often regarded as being easier to study than other jet propulsion fuels in that it is essentially a single-component jet fuel, making it a favorable target for kinetic modeling. Component analyses indicate that JP-10 consists of approximately $96.5 \mathrm{w} \%$ exotetrahydrodicyclopentadiene, $1.0 \mathrm{w} \%$ adamantane and $2.5 \mathrm{w} \%$ endo-tetrahydrodicyclopentadiene (Figure 1). Due to the complexity of the molecules involved, we assume in the remainder of this work that JP-10 can be regarded as its primary component. 

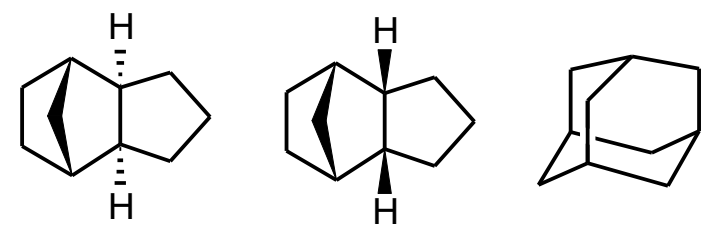

Figure 1. Main constituents of JP-10: (from left to right) exo-tetrahydrodicyclopentadiene, endotetrahydrodicyclopentadiene and adamantane.

The current literature data provide an incomplete picture of JP-10's complex pyrolysis and combustion behavior. Moreover, available datasets often show significant discrepancies amongst each other. One of the first extensive studies on the pyrolysis of JP10 was conducted by Davidson et al. ${ }^{2}$ using high speed UV adsorption behind reflected shock waves at $1400 \mathrm{~K}$ and 1 atm. The authors identified cyclopentene as one of the major initial decomposition products amongst other major decomposition products: ethene, propylene, benzene, butadiene and cyclopentadiene. Nakra et al. ${ }^{3}$ studied the pyrolysis of JP-10 in a micro flow tube reactor at roughly $2 \times 10^{-3} \mathrm{~atm}$ with electron impact and ion impact mass spectra analysis. Their studies covered high temperature pyrolysis and millisecond residence times but did not include the highpressure regime. Benzene and cyclopentadiene were identified as main decomposition products but the authors failed to identify large concentrations of cyclopentene, which would be expected from the Davidson et al. experiments. Striebich and Lawrence ${ }^{4}$ focused on higher pressure experiments, working at supercritical conditions of 34 atm and 373-873 $\mathrm{K}$ to illustrate the thermal stability of JP-10, which was found to be more stable than other jet fuels tested. They found that JP-10's decomposition products were predominantly alkanes and alkenes, but they also detected smaller concentrations of cyclohexene and dimethylcyclopentadiene. Xing et al. ${ }^{5}$ studied the decomposition of JP-10 at $900 \mathrm{~K}$ and pressures ranging from 1 to $40 \mathrm{~atm}$, contributing to an enhanced understanding of reaction pressure on product selectivities. That work points to secondary reactions of alkenes, formed during the initial decomposition phase, as the major contributing pathways to aromatic species formation. However, this conclusion contradicts Herbinet et al.'s observation that some aromatics such as benzene and toluene must also have direct reaction channels, based on experiments conducted in a jet stirred reactor. ${ }^{6}$

The combustion of JP-10 has primarily been studied under conditions that mimic those applicable in engines: residence times are typically very short (i.e. ms range) with temperatures exceeding $1000 \mathrm{~K}$. These studies generally focused on measuring ignition delays and do not report detailed product distributions, which are important for understanding engine performance and predicting the fuel's sooting behavior. Davidson et al. ${ }^{7}$ reported ignition delay times and $\mathrm{OH}$ concentration time histories behind reflected shock waves in the temperature range $1300-1700$ $\mathrm{K}$ with pressures ranging from 1-6 atm. Colket and Spadacinni ${ }^{8}$ reported ignition delays in the temperature range $1250-1500 \mathrm{~K}$. Mikolaitis et al. ${ }^{9}$ conducted ignition delay measurements at higher temperatures up to $2000 \mathrm{~K}$ and higher pressures of $10-25 \mathrm{~atm}$. They also found that JP10 's ignition delays were generally insensitive to fuel additives. More recently, Wang et al. ${ }^{10}$ 
reassessed ignition delays for JP-10 at pressures of 1.5 to 5.5 atm and temperatures ranging from 1000 to $2100 \mathrm{~K}$ to fill gaps between the other datasets.

Many computational studies have focused on solving specific problems related to understanding JP-10's decomposition chemistry. Zehe and Jaffe ${ }^{11}$ studied the thermochemistry of exo-tetrahydrodicyclopentadiene using composite methods such as CBS-QB3 and the Gx family of methods. Bozzelli et al. ${ }^{12}$ extended this to all possible radicals formed after abstracting a hydrogen from JP-10 and used a reaction scheme approach to obtain enthalpies of formation for various radicals with approximately $1 \mathrm{kcal} \mathrm{mol}^{-1}$ accuracy. Chenoweth et al. ${ }^{13}$ used a reactive force field (ReaxFF) to identify major initial decomposition pathways including (a) a path leading to the formation of ethene and a C8 hydrocarbon and (b) a path producing two C5 hydrocarbons. Various reactions paths have also been studied by Yue et al. ${ }^{14}$ using the M06-2X functional. Recent work by Magoon et al. ${ }^{15}$ focused on calculating energy barriers for intramolecular disproportionation reactions that are important during the pyrolysis of JP-10.

Despite the broad range of experimental and computational studies that have been conducted in an effort to increase our understanding of JP-10's decomposition chemistry, the development of an accurate, detailed combustion mechanism has remained elusive. The pyrolysis mechanism developed by Herbinet et al. ${ }^{6}$ lacks oxidation chemistry and is not publicly available. The first JP-10 combustion mechanism was developed by Li and coworkers ${ }^{16}$ a decade ago; however, the initial decomposition steps were modeled using lumped reactions to capture a simple model which largely avoided the kinetics of any species larger than $\mathrm{C}_{3}$. The resulting lumped model contained 36 species and 174 reactions, with no decomposition products larger than $\mathrm{C}_{5}$. This preliminary model was admittedly highly speculative for kinetics involving species larger than $\mathrm{C}_{3}$ but yielded satisfactory ignition delay predictions relative to available shock tube data. For many years, the Li et al. mechanism was, to our knowledge, the only published mechanism available for modeling JP-10 combustion.

Authors of the present paper developed and refined the first detailed JP-10 combustion mechanism ${ }^{15}$ using RMG (Reaction Mechanism Generator), ${ }^{17}$ an open-source software package for automatically building detailed kinetic mechanisms. This first-generation mechanism characterized the initial decomposition kinetics of JP-10 combustion but included very rough estimates for many important reactions and had little experimental validation due to lack of speciation data. More recently, Vandewiele et al. ${ }^{18}$ generated an improved pyrolysis model validated by new experimental data obtained at Ghent University.

In this paper, RMG was used to generate a new comprehensive combustion model for JP-10, extending on the Vandewiele pyrolysis model with updates to rate coefficients and thermochemistry based on quantum chemistry calculations and validated through new shock tube experiments. The aim of this new set of experiments is to fill in the gap existing in the current literature and better understand JP-10's decomposition and oxidation chemistry. Products of decomposition were identified and measured behind reflected shock waves at pyrolysis and pre- 
ignition oxidation conditions ranging from very lean $(\Phi=0.14)$, lean $(\Phi=0.24)$, to stoichiometric $(\Phi=1.0)$. The experiments were conducted at temperatures ranging between 1000 and $1600 \mathrm{~K}$, pressures of 6-8 atm, and a residence time of about $500 \mu \mathrm{s}$. These data were further used to validate a new detailed kinetic model generated by RMG that captures both the pyrolysis and oxidation chemistry of JP-10. Modeling advances were accomplished by supplementing the RMG database with higher fidelity computational estimates and reported literature values for critical kinetics and thermodynamic parameters, in addition to algorithmic improvements to the RMG software. This work highlights the current capabilities of computerbased kinetic model generation with respect to cyclic chemistries such as JP-10 as well as opportunities for further research.

\section{Methodology}

\section{Shock tube experiments}

The JP-10 pyrolysis and oxidation experiments were carried out in a $2.54 \mathrm{~cm}$ internal diameter pressure-driven single-pulse shock tube, of the design described by Lifshitz et al. (Figure 2) ${ }^{19}$ The driven (sample) section is $1.75 \mathrm{~m}$ long, and the driver section (adjustable for "tuning") is $0.91 \mathrm{~m}$ long. A Mylar diaphragm, $2.0 \mu \mathrm{m}$ thick, separates the two sections. A $3.2 \times 10^{-2} \mathrm{~m}^{3}$ dump tank is positioned just downstream from the diaphragm for the purpose of preventing multiple reflections of the shock. Two rapid-response pressure sensors are mounted in the wall of the driven section to detect the arrival of the incident and reflected shocks; the output is amplified and recorded on digitizing storage oscilloscopes, and reaction temperatures are determined from the recorded shock velocities.

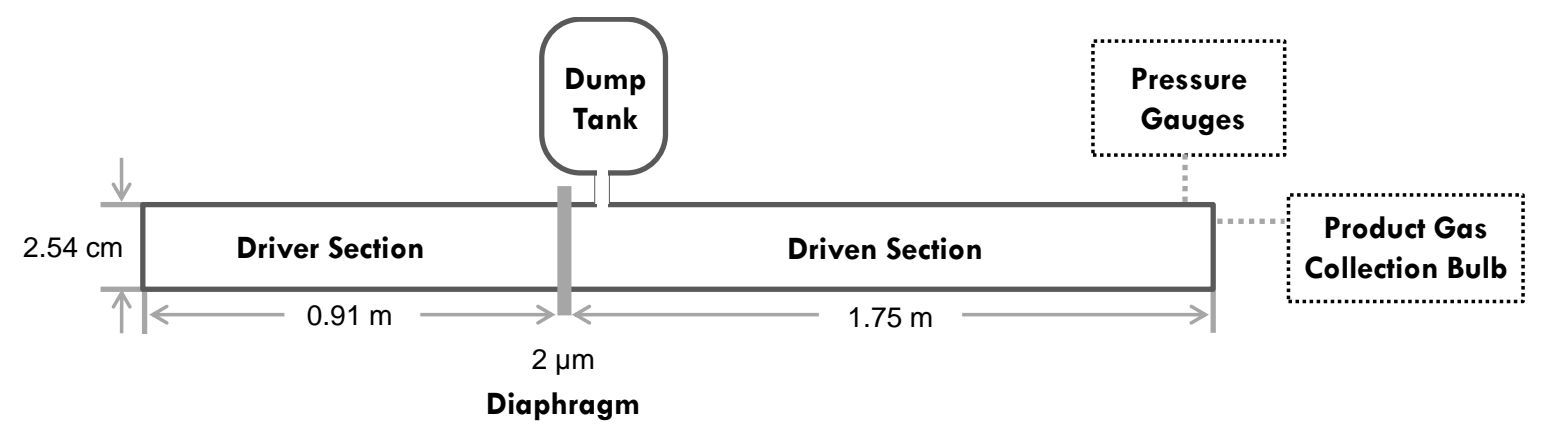

Figure 2. Schematic of the shock tube used for the experimental study.

Before each experiment, the entire system is evacuated by a molecular drag pump that is backed by a diaphragm pump, to a pressure $<0.1 \mathrm{~Pa}$. Helium is used as the driver gas, and research-grade argon is used as the diluent for the reactant samples. Reactant samples were prepared in $1.0 \mathrm{~L}$ glass bulbs (sealed with Teflon stopcocks) containing glass beads to enhance mixing. A few drops of liquid JP-10 were added, and the bulb was evacuated briefly (some liquid JP-10 remained). After this, the bulb was filled to 850 Torr with either argon (for pyrolysis) or a 
pre-mixed $\mathrm{O}_{2} / \mathrm{Ar}$ mixture (for oxidation) and swirled for at least 5 minutes to establish a saturated JP-10/Ar or JP-10/O $/ 2$ Ar mixture. Reactant samples were stored for at least an hour at $20^{\circ} \mathrm{C}$ before use. Heated tubes $\left(\sim 50^{\circ} \mathrm{C}\right)$ connecting the sample bulb and shock-tube prevent JP-10 condensation, and $2.5 \times 10^{4}$ to $5.0 \times 10^{4} \mathrm{~Pa}$ of the reactant sample was eventually transferred to the shock-tube. As soon as the desired sample pressure had been measured in the shock tube the shock was initiated by exceeding the diaphragm's bursting pressure with the He driver gas. Immediately after each shock, the product sample was withdrawn through a small outlet in the side-wall $7.5 \mathrm{~mm}$ upstream of the end wall of the driven section into a $100 \mathrm{~cm}^{3}$ glass bulb that had been previously evacuated and then filled with a small amount $\left(1.3 \times 10^{3} \mathrm{~Pa}\right)$ of a calibration mixture containing argon and measured amounts of two standards, isobutene and ciscyclooctene. These two compounds were chosen as calibration standards since neither had been detected among the JP-10 pyrolysis or oxidation products in earlier qualitative test runs, and because both were well-resolved from the pyrolysis and oxidation products.

The gas collection bulbs containing product gas samples and the calibration standards were analyzed on two GC-MS systems: an Agilent 6890 GC with an Agilent 7850 MS and a HewlettPackard 5890 GC with a Hewlett-Packard 7820 MS. The Agilent GC-MS was used to analyze compounds greater or equal to $\mathrm{C}_{5}$ with an Agilent HP5-MS (5\% Phenyl Methyl Siloxane) column of dimensions $30 \mathrm{~m} \times 0.25 \mathrm{~mm} \times 0.25 \mu \mathrm{m}$. The HP GC analyzed compounds smaller than $\mathrm{C}_{5}$ using a Restek PLOT S-Bond column of dimensions $30 \mathrm{~m} \times 0.25 \mathrm{~mm} \times 0.53 \mu \mathrm{m}$. Previously, a set of 10 compounds of known concentration, ranging from C3 to C10 in size, had been analyzed and quantified on each GC-MS. Using calibration analyses of cis-cyclooctene, isobutene and the ten other compounds, standard curves were made to relate the MS response to the molar concentration of each species. This gave a set of MS response calibration curves that were offset according to molecular weight and molecular structure. For species found in the JP10 reaction products that were specifically calibrated for with standards, the product quantification was done based on direct response factors for those specific compounds. For species found in the reaction products that were not in the calibration standards, the MS response factors were interpolated from the standard curves for species of similar molecular weight and structure. In all cases the peak areas were first normalized by the MS response to ciscyclooctene or isobutene in order to correct for day-to-day variations in the responses of the two GC-MS instruments. Every sample was analyzed three times before taking an average mole fraction of each species.

Under pyrolysis and stoichiometric oxidation conditions, carbon balances of 80 to $110 \%$ were obtained. This is within the accepted error in GC-MS quantitative analysis, i.e. +/- 20\%, and averages on the low side of $100 \%$ mainly due to our inability to detect $\mathrm{CO}, \mathrm{CO} 2, \mathrm{CH} 4$ and $\mathrm{HCHO}$ because of the peaks' overlapping retention times with the diluent gas Ar. Due to the excess argon in the samples, it was very difficult to detect and quantify most of the lighter species, including all $\mathrm{C} 1$ compounds. Under leaner conditions, carbon balances based on the 
species we were able to quantify dropped to approximately $50 \%$, due to larger relative amounts of $\mathrm{CO}$ and $\mathrm{CO}_{2}$ formation.

The pressure, temperature and density of the reacting shock-heated gas immediately after passage of the reflected shock was calculated from measured incident shock speeds, the initial (pre-shock) sample temperature and pressure, conservation equations (mass, momentum, energy), and heat capacity polynomials for JP-10, $\mathrm{O}_{2}$ and Ar. The reaction time for the sample was determined from the pressure versus time traces at the downstream end of the tube, as the time interval between initiation of the reflected shock and arrival of the cooling expansion wave. This time is longer for gas at the end of the tube, but is less for gas that was further upstream. Assuming that the composition of each $100 \mathrm{~cm}^{3}$ product sample collected is representative for the product gas at a certain position in the shock tube, we can derive the average reaction time from its average location under the compressed reaction conditions. Samples had an effective average reaction time of $500(+/-10 \%) \mu \mathrm{s}$.

\section{Experimental error analysis}

A single-pulse shock tube can be, in many respects, an ideal choice for the study of rapid thermally-induced reactions under homogeneous conditions. Gas samples can be brought rapidly $\left(\mathrm{t} \sim 10^{-11} \mathrm{~s}\right)$ and homogeneously to temperatures ranging from hundreds to thousands of degrees above ambient temperature, at pressures ranging from $<10^{3}$ to $>10^{7} \mathrm{~Pa}$, then quenched back to room temperature and recovered for analysis. Since the heating is brought about by the passage of a compression shock wave through the gas sample, the walls of the apparatus remain essentially at room temperature throughout the sub-millisecond reaction time; a thin boundary layer of cold gas forms along the walls insulating them from the hot reacting gas. This effectively eliminates the potential complications of catalytic surfaces but also means that some of the recovered gas for analysis was not heated to the temperature of the gas in the center of the tube. In the present study, that percentage was determined by running JP-10/Ar samples to temperatures above $1600 \mathrm{~K}$, under which JP-10 consumption should have been close to $100 \%$. These experiments indicated that the actual residual reactant concentration amounted to approximately $1 / 3$ of the original JP-10 feed (i.e. $67 \% \pm 5 \%$ of the feed reacted).

Another limitation arises because of the inherent differences between compression (heating) and expansion (cooling) waves. In an ideal T-jump experiment, both the heating and the cooling should be instantaneous. Shock waves are by nature convergent processes; as the shock front is formed, successive stages of compression travel through increasingly hot gas, at progressively faster velocities, until they coalesce into a single, abrupt pressure discontinuity. Expansion waves travel through progressively cooler gas and therefore diverge. Thus the desired instantaneous heating of the reactant sample is achieved, but the cooling of the reaction products at the end of the reaction time is not instantaneous. In the shock tube used in this study, the typical initial

cooling rate was determined, from the recorded pressure trace, to be $>5 \times 10^{5}{ }^{\circ} \mathrm{C} / \mathrm{s}$; so $100 \mu \mathrm{s}$ into the cooling process (one fifth of the reaction time) the temperature drops at least 50 degrees. 
The rate at which each reaction is quenched depends on the activation energy and the reaction order (since the gas density also drops, lowering collision rates). For the initial pyrolysis reactions of JP-10, with estimated activation energies $>80 \mathrm{kcal} / \mathrm{mol}$, a temperature decrease from 1200 to $1150 \mathrm{~K}$ should lower the rate of JP-10 consumption by more than a factor of four. However, abstraction or addition reactions of radicals, such as would form alkenes, alkynes and aromatic species, will be slowed down less rapidly. By the time the product sample is collected for analysis, it will contain only neutral, non-radical species. Thus, the concentrations of benzene, toluene, cyclopentene, cyclopentadiene and similar product species are likely to be greater than they were in the shock tube at the end of the reaction time but before the cooling process occurred. Considering all these uncertainties, the total estimated uncertainty in species concentrations is approximately $30 \%$. For this reason, we believe that the pyrolysis and oxidation experiments performed in this study are most useful for qualitative and semi-quantitative comparisons with the model.

The final source of uncertainty in the experimental data is the error associated with the reaction temperature, which is back-calculated from the recorded shock wave velocities. These times are determined from the pressure-jump found in the oscilloscope readings, which have an uncertainty of approximately $0.4 \mu \mathrm{s}$ (the oscilloscopes have a resolution of $0.2 \mu \mathrm{s}$ ). The temperature error arises from the propagation of the oscilloscope error and from the assumption of complete translational, rotational, and vibrational equilibration of kinetic energy when converting shock speed to temperature. We estimate the total temperature uncertainty to be about $3-4 \%$, or $50 \mathrm{~K}$. This is a significant uncertainty, since the chemistry can vary greatly with a $50 \mathrm{~K}$ change in temperature.

\section{Detailed chemical kinetic modeling}

RMG was used to generate the kinetic models in JP-10 combustion and pyrolysis by automatically discovering chemical species and elementary reactions involved. Rate constants and thermodynamic parameters for the species and reactions are based on an integrated database of libraries and estimation rules. RMG constructs a kinetic mechanism by selecting the relevant subset of possible species and reactions with significant fluxes at the user's specified reaction conditions. This automated procedure for kinetic model generation is designed to avoid the process of constructing kinetic models manually, which can be very tedious and error-prone for complex chemistries such as JP-10 decomposition. However, it must be noted that automated mechanism construction is an actively evolving research area that continuously incorporates new improvements from the investigation of increasingly complex kinetics systems. The characterization of JP-10 represents the first time RMG has been used to investigate the combustion kinetics of a fused cyclic molecule. The model development process led to multiple 
improvements to RMG's databases. These refinements were based on sensitivity analysis and comparison with experiment to identify the species and reactions whose thermodynamics and kinetics mostly strongly influence the model predictions. All parameter refinements have a justifiable basis in literature, ab initio calculations, accepted estimation procedures, or physics.

The details of RMG methods and operation are available in previous works ${ }^{17,20}$ and are briefly summarized here. RMG is operated by specifying an initial set of reactants and reaction conditions (temperature, pressure and species compositions), as well as a reaction system termination time or conversion of interest. Once these conditions are specified, the process of building the kinetic model occurs via a rate-based algorithm ${ }^{20 \mathrm{c}}$ (Figure 3): RMG reacts the initial species in all possible ways according to known reaction family templates and performs a timeintegration of the kinetics equations for an isothermal, isobaric batch reactor. After each integration time step, RMG evaluates the rate at which each new chemical species (called an "edge" species) is produced. The total reaction flux of all species within the core is defined as the characteristic flux $R_{\text {char }}$. If a new "edge" species is produced with a flux greater than $\varepsilon R_{\text {char }}$, where $\varepsilon$ is a user-specified error tolerance, that species is added to the mechanism "core," including all the reactions producing that species. This new core species is then reacted with all other core species in the mechanism to generate a new set of edge species and reactions. The time-integration restarts using the updated core and the expanded list of edge species is again examined for significant species to be added to the core. The process continues, incrementally growing the mechanism with each iteration until the user-specified end conditions are satisfied.

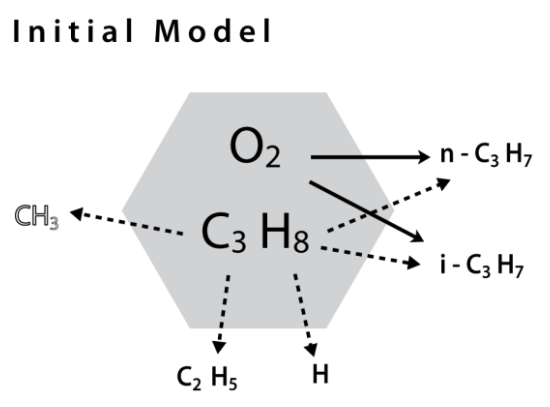

AFTER Second Iteration
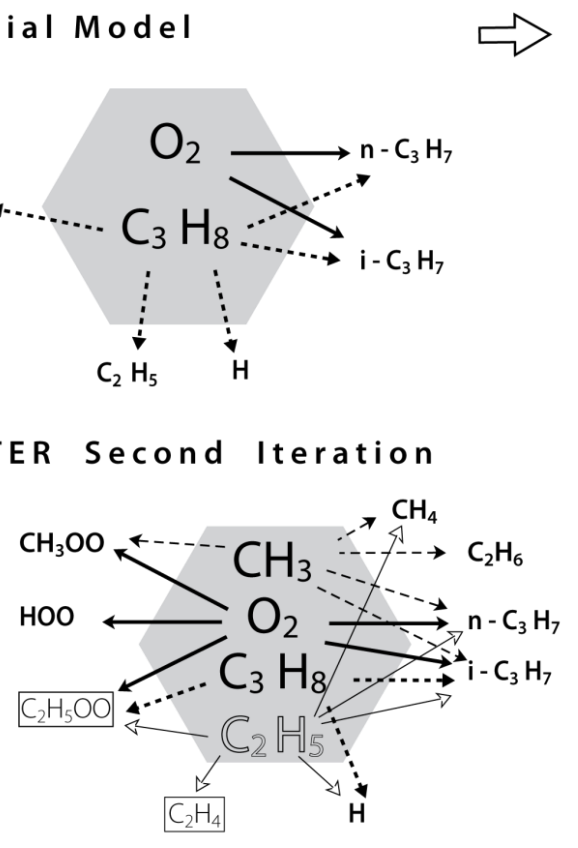

AFTER First Iteration
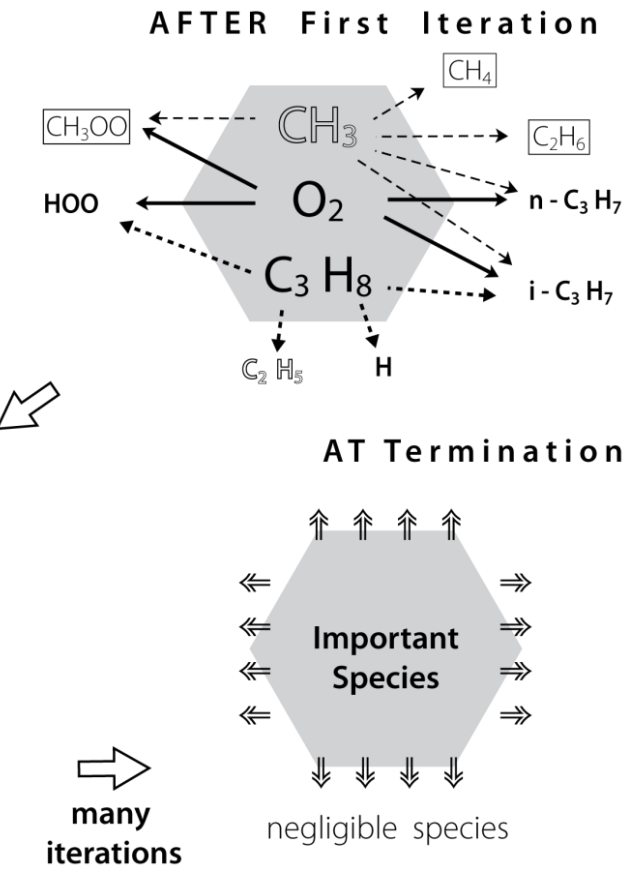

Figure 3. RMG automatic mechanism construction illustrated for propane oxidation. The process begins with an initial set of reactions in the mechanism "core" (inside the shaded area ). After each iteration, 1) new "edge" species (outside the shaded area) are generated based on the possible reactions of the core and 2) a 
new species is selected from the edge, for addition to the core. The core is expanded over many iterations until the user-specified end criteria are satisfied.

\section{Model generation}

The final JP-10 combustion model presented in this work was obtained by merging four smaller networks generated at the reaction conditions of interest. The final model contains 691 species and 15518 reactions. A low temperature pyrolysis model was generated to cover the reaction conditions associated with the Ghent pyrolysis data. Medium temperature pyrolysis and oxidation models were generated to cover the reaction conditions of the shock tube data presented in this study. Finally, a high temperature oxidation model was generated to span the range of experimental conditions associated with the ignition delays. The conditions associated with these four models span a broad range of temperatures, pressures and equivalence ratios as detailed in Table 1.

Table 1. RMG model generation conditions for the four subnetworks in the JP-10 mechanism.

\begin{tabular}{|c|c|c|c|}
\hline Network & $\begin{array}{c}\text { Equivalence Ratio } \\
(\mathbf{\Phi})\end{array}$ & $\begin{array}{c}\text { Temperature } \\
(\mathbf{K})\end{array}$ & Pressure (atm) \\
\hline Low temperature pyrolysis & - & 1000 & 1.7 \\
\hline Medium temperature pyrolysis & - & 1400 & 7 \\
\hline Medium temperature oxidation* & 0.14 & 1400 & 7 \\
\hline High temperature oxidation & 0.14 & 2000 & 1 and 20 \\
\hline
\end{tabular}

* pre-ignition

For all models, the JP-10 goal conversion was set to $99 \%$ with an error tolerance of $\varepsilon=0.05$. RMG reached full convergence under all simulated reaction conditions. In order to control the radical addition reactions proceeding to much larger hydrocarbons, all jobs restricted the maximum number of carbon atoms per species to 10 , the number of heavy atoms per species to 12 , and the number of unpaired electrons to 2 or less. All networks were generated within the high pressure limit; however, rates for small molecule reactions involving $\mathrm{OH}, \mathrm{H}, \mathrm{H}_{2} \mathrm{O}, \mathrm{CO}$, etc. which are highly influential and strongly pressure dependent were taken from the EFRC v0.3 mechanism $^{21}$ and seeded into the RMG-generated models. RMG also had access to a set of calculated rate coefficients (mainly CBS-QB3 ${ }^{22}$ and BMK/6-311G(2d,d,p) ${ }^{23}$, see Supporting Information). The calculated reactions are mainly hydrogen abstraction reactions from JP-10 and $\beta$-scissions in the most important JP-10 radicals formed after hydrogen abstraction from JP-10. For the hydrogen abstraction reactions, the 1-D hindered rotor (1D-HR) approach was used to obtain a more accurate treatment of low vibrational internal rotations. The methodology for this approach has been described in the work of Sharma et al. ${ }^{24}$ Due to their importance in the initial decomposition steps, rate coefficients for intramolecular disproportionation reactions presented in the work of Magoon et al. ${ }^{15}$ calculated using various multireference methods were also included in this database. 
Compared to previous models this current model benefits from major upgrades to the RMG thermochemical database which are (a) revision of the ring strain corrections for polycyclic compounds, (b) improved databases for hydrogen abstraction reactions and $\beta$-scission reactions that use rate rule estimates based on the group additivity concept for Arrhenius parameters ${ }^{25}$ and (c) updated estimates for important intramolecular reactions. RMG traditionally estimates thermodynamic quantities using a Benson group additivity scheme, which is unable to deal with many cyclic and fused cyclic compounds accurately without requiring a large number of ad hoc ring corrections. To avoid inaccuracies, when an exact polycyclic ring correction cannot be found, we used the on-the-fly quantum mechanics (QM) feature ${ }^{26}$ within RMG to perform quantum calculations for cyclic species based on estimating explicit 3D geometries. In particular, on-the-fly semi-empirical PM7 calculations were performed for cyclic species using MOPAC2012. ${ }^{27} \mathrm{RDKit}^{28}{ }^{28}$ OpenBabel, ${ }^{29} \mathrm{cclib}^{30}{ }^{30} \mathrm{InChI},{ }^{31}$ and SYMMETRY ${ }^{32}$ software packages were also used in this process. For cyclic radicals, PM7 calculations were performed for the parent saturated molecule and hydrogen bond increments ${ }^{33}$ were applied. This approach is described in further detail elsewhere. ${ }^{26}$

After model generation, additional third body reactions involving $\mathrm{Ar}$ and $\mathrm{Ne}$ were added manually as RMG currently cannot deal with them. These reactions involve recombination reactions of atoms such as $\mathrm{H}$ and $\mathrm{O}$. The initial model however tended to over-predict the JP10 's decomposition rate and yielded too much propene. A sensitivity analysis showed that this was likely caused by an over-prediction of the rate coefficient for hydrogen abstraction reactions from JP-10 by allyl estimated by group additivity. ${ }^{25 b}$ To correct for this we lowered the $n$ factor in the three parameter Arrhenius equation for hydrogen abstraction reactions from JP-10 by allyl radical from 4.24 to 4.00 .

\section{Results and discussion}

\section{The reaction network}

The pyrolysis of JP-10 can be understood as a three step process. In the initial step, JP-10 isomerizes to other components, typically by a ring opening followed by an intramolecular disproportionation. The model predicts in this initial stage the isomerization of JP-10 to 2cyclopentylcyclopentene (2CC) and 4-ethylbicyclo[3.3.0]oct-2-ene (4EBO), as shown in Figure 4. The shock tube experiments confirm the presence of $2 \mathrm{CC}$ during pyrolysis and oxidation (see Supporting Information). This is also supported by experimental data presented by Vandewiele et al. ${ }^{34}$, where many unidentified $\mathrm{C}_{9}$ and $\mathrm{C}_{10}$ species with similar volatility to JP-10 were found at low conversion. The species undergo bond scissions which lead to the formation of two radicals that initiate the radical mechanism. Those radicals undergo $\beta$-scissions until a radical is obtained that has a sufficiently long lifetime to participate in hydrogen abstraction reactions. In agreement 
with Herbinet et al., ${ }^{6}$ we find that the main radical initiation reaction is bond scission in 2cyclopentylcyclopentene (2CC in Figure 4) with the formation of cyclopentenyl and cyclopentyl radicals. Cyclopentenyl radicals subsequently decompose by $\beta$-scission to cyclopentadiene and hydrogen radicals. Cyclopentyl radicals decompose mainly by ring opening and $\beta$-scission to ethene and allyl.

Radical chain reactions constitute the second step of the decomposition process, which begins with hydrogen abstractions from JP-10. Allyl and $\mathrm{H}$ radicals formed in step 1 are responsible for most of the hydrogen abstraction reactions, which can occur at 6 different sites on JP-10. As intramolecular hydrogen abstraction reactions in JP-10 radicals are strongly hampered due to its fused ring structure, each primary hydrogen abstraction reaction initiates a distinct decomposition channel with its own set of products. The main decomposition products for the four dominant channels are presented in Figure 4. It can be seen that the main products are ethene, 1,3-cyclohexadiene, cyclopentadiene and propene. Once a sufficient amount of products are formed, secondary reactions will lead to the formation of aromatic components from the main decomposition products. This can be seen as an important third step in the pyrolysis mechanism and is often regarded one of the most difficult ones to model. Under oxidation conditions, the decomposition of JP-10 will be very similar to the one observed during the pyrolysis, except that oxygen will participate in chain branching reactions, speeding up the radical reactions and reacting with the pyrolysis products to form $\mathrm{CO}, \mathrm{H}_{2} \mathrm{O}$ and $\mathrm{CO}_{2}$. 


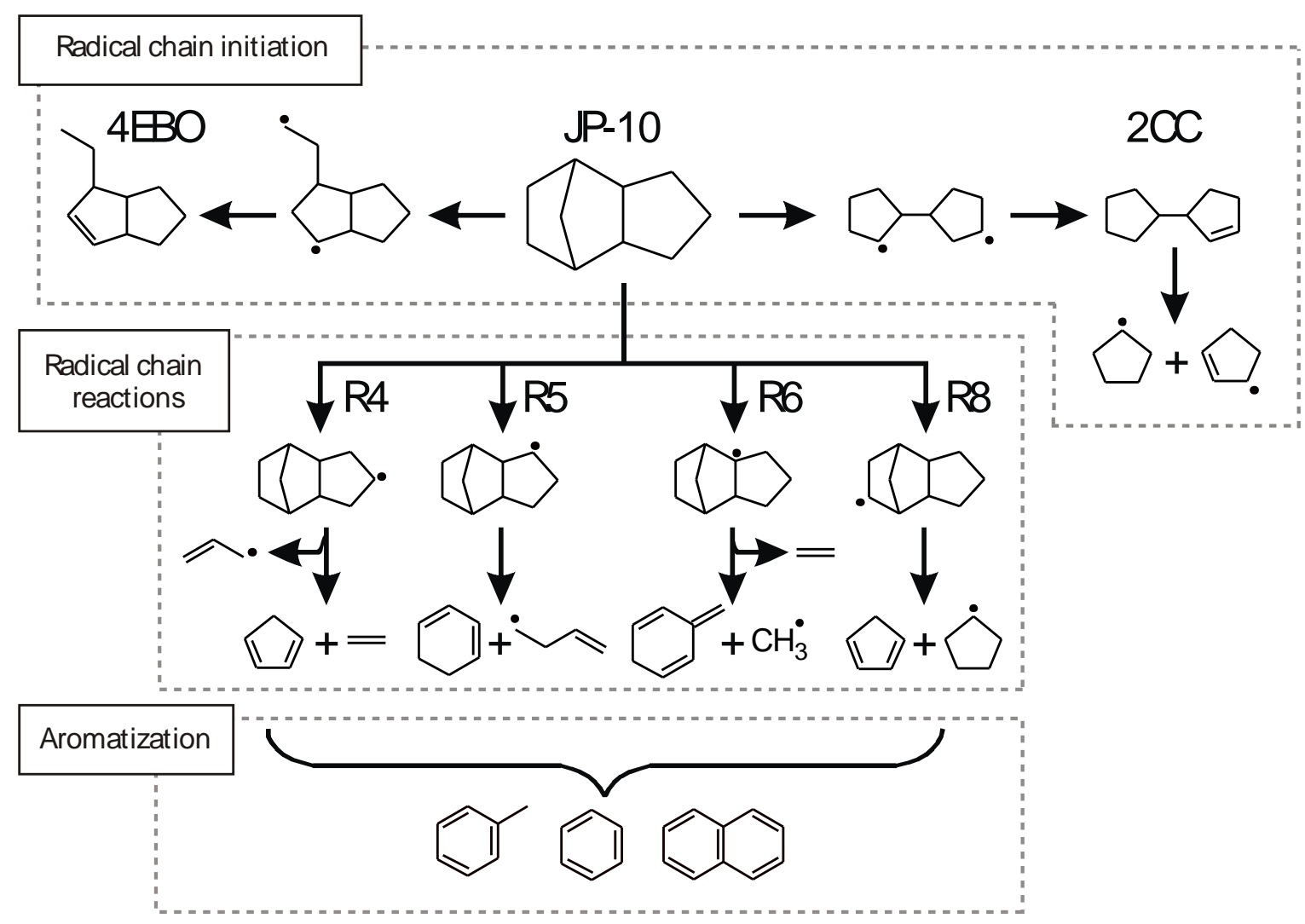

Figure 4. The three step mechanism of JP-10 thermal decomposition: radical chain initiation reactions (top), radical chain reactions (middle), and finally aromatization (bottom).

In Figure 5 the predicted decomposition pathways for the pyrolysis and stoichiometric oxidation of JP-10 is shown. Both conditions for the model were simulated in a plug flow reactor operated at $1000 \mathrm{~K}$ and a pressure of $1.7 \mathrm{~atm}$. At $10 \%$ conversion, almost $8 \%$ of the JP-10 decomposes by ring opening reactions leading to three different biradicals (BR1, BR2, and BR3 in Figure 5). These biradicals undergo intramolecular disproportionation reactions leading to saturated products that further decompose to two radicals each. Shown in Figure 5, BR1 leads to the formation of two cyclic $\mathrm{C}_{5}$ radicals that further react to form cyclopentadiene and hydrogen radicals or allyl and ethene. BR3 has a decomposition path leading to the formation of ethyl and a bicyclo[3.3.0]oct-2-enyl radical. Approximately 92\% of the decomposition of JP-10 is governed by hydrogen abstraction reactions at the pyrolysis conditions. The main abstractors are the hydrogen radical and allyl, each accounting for approximately one fourth of the total decomposition rate of JP-10. The other main abstractors from JP-10 are the methyl radical, phenyl and cyclopent-2-enyl. The favored abstraction site is the secondary carbon atom of the norbornane-type ring structure forming $\mathrm{R} 8$ in Figure 5. R8 decomposes mainly to cyclopentadiene and cyclopentyl. Hydrogen abstractions leading to R4, R5 and R6 are nearly equally important. R4 decomposes to an allyl radical and norbornene, which then decomposes by a reverse Diels-Alder reaction to cyclopentadiene plus ethene. This reaction path has also been 
proposed by Herbinet et al. ${ }^{6}$ However, the reaction network developed by RMG predicts alternative decomposition channels for radicals R5 and R6. Almost 50\% of the R5 radicals form benzene, by (a) a path that leads to the formation of butadiene and cyclohexenyl through a bicyclo(3.1.0)hexane ring intermediate or (b) a path that leads to the formation of two ethene molecules and cyclohexadienyl that involves the formation of intermediate tricyclo[4.2.1.1(2,5)]decyl radicals. Channel (a) is favored at higher temperatures while at lower temperatures $(<1000 \mathrm{~K})$ channel $(\mathrm{b})$ is dominant. The rate of production analysis further shows that R6 primarily decomposes to toluene or important toluene precursors such as 3methylidenecyclohexa-1,4-diene.

Under stoichiometric oxidation conditions at $1.7 \mathrm{~atm}$ and $1000 \mathrm{~K}$, it was found that at $10 \%$ conversion, ring opening reactions contribute to a mere $0.4 \%$ of the total consumption rate of JP10. This is almost a factor of 20 lower than observed for pyrolysis. Chain branching reactions, particularly $\mathrm{H}+\mathrm{O}_{2} \rightarrow \mathrm{OH}+\mathrm{O}$ and $\mathrm{H}_{2} \mathrm{O}_{2} \rightarrow \mathrm{OH}+\mathrm{OH}$, lead to an increase in the radical count and thus a faster radical mechanism. Abstractions by $\mathrm{OH}$ and $\mathrm{OOH}$ account for almost $80 \%$ of the total consumption rate of JP-10. Hydrogen abstractions predominantly occur from secondary carbon sites in JP-10, leading to alkyl radicals R4, R5, and R8. In contrast to pyrolysis, hydrogen abstraction reactions from the tertiary carbon atom of the norbornane structure become important and lead to a significant reaction channel for $\mathrm{R} 7$ formation. This channel accounts for almost $14 \%$ of JP-10's decomposition under stoichiometric combustion whereas it contributes to only $3 \%$ of the consumption in pyrolysis. R7 primarily decomposes through beta-scissions to butadiene and $\mathrm{C}_{6}$ products passing through an intermediate 9-membered ring structure.

RMG identified reaction paths to the following aromatic products: benzene, toluene, ethylbenzene, styrene and indene. As shown in Figure 5, a large part of R5 decomposes to cyclohexenyl and cyclohexadienyl radicals, which can easily dehydrogenate to form benzene. Most of the benzene (approximately $70 \%$ at $1000 \mathrm{~K}, 1.7 \mathrm{~atm}$ and a conversion of $10 \%$ ) is hence formed by the $\beta$-scission reaction in cyclohexadienyl leading to benzene and a hydrogen atom. Other reactions leading to the formation of benzene are well-skipping reactions as studied by Sharma et al. ${ }^{35}$ Approximately $25 \%$ of the benzene is computed to be formed by well-skipping reactions involving fulvene:

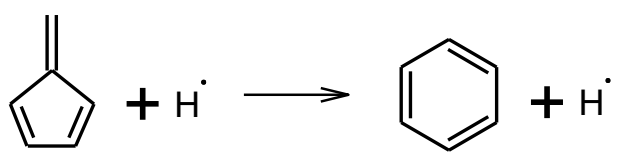

$(\mathrm{RXN}-1)$

The formation of fulvene is initiated by the recombination reaction of a methyl radical with cyclopentadienyl, which forms methylcyclopentadiene. Hydrogen abstraction reactions from this component followed by $\beta$-scission/disproportionation reactions yield fulvene. Toluene is predominantly formed by decomposition of R6 (see Figure 5). The model predicts that approximately $50 \%$ of the toluene is formed by $\beta$-scission reactions of dimethylcyclohexadienyl (DMC in Figure 5): 


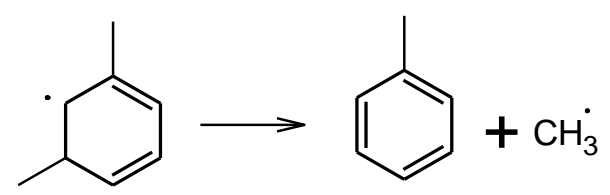

$(\mathrm{RXN}-2)$

The other 50\% is formed by hydrogen abstraction reactions involving benzyl radicals, which are formed by hydrogen abstraction from 3-methylidenecyclohexa-1,4-diene, another decomposition product of R6. As benzyl is fairly abundant in the system, the main reaction path leading to ethylbenzene is the recombination of benzyl with methyl radical. Ethylbenzene easily dehydrogenates and forms styrene in successive reactions. Indene is predominantly formed from a path initiated by the addition of cyclopentadienyl to cyclopentadiene (RXN-3). This model uses the rate coefficient for this well-skipping reaction computed by Cavallotti et al. ${ }^{36}$

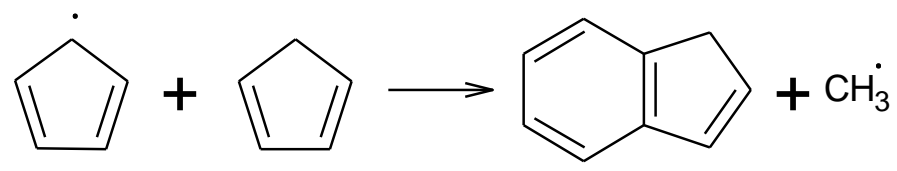

$(\mathrm{RXN}-3)$

The reaction network presented here can be regarded as an extension of the Vandewiele et al. ${ }^{18}$ JP-10 pyrolysis model to include combustion. Due to the scarcity of rate rules in certain reaction families in the older version of RMG, Vandewiele et al. opted not to include intramolecular hydrogen abstraction reactions and exo- and endo-cycloadditions. The new network reported here includes these reaction families with the addition of new rate rules and additional updates to the thermo and kinetics databases. As shown in Figure 5, these reaction families are important in the paths towards toluene and benzene formation. The inclusion of these pathways corrects for the underestimation of ethene, coproduct in the formation of benzene, and overestimation of cyclopentadiene yields in the Vandewiele et al. mechanism. 


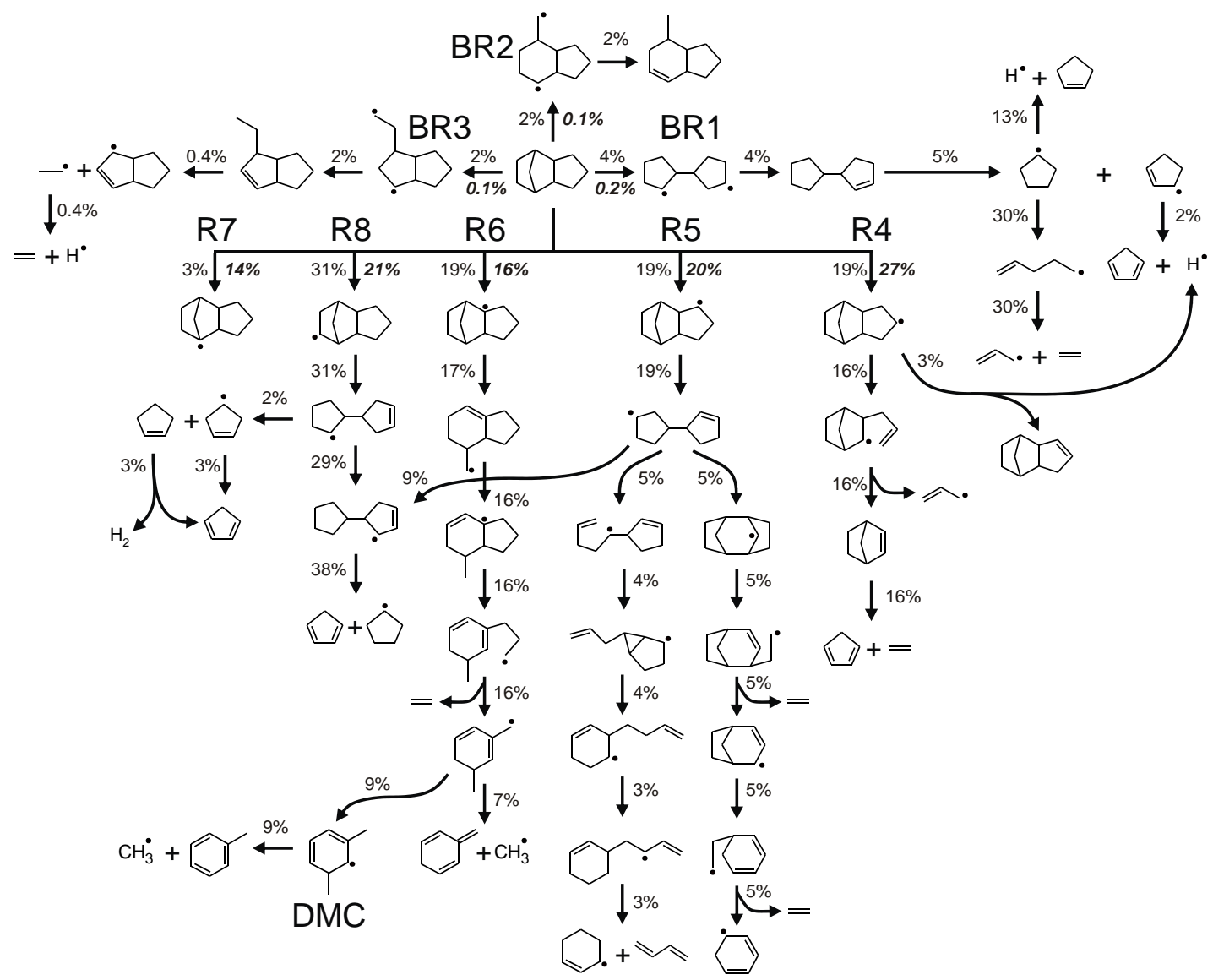

Figure 5. Predicted major decomposition pathways of JP-10 pyrolysis $\left(9.34 \mathrm{~mol} \% \mathrm{JP}-10\right.$ in $\left.\mathrm{N}_{2}\right)$ and stoichiometric combustion $\left(0.62 \mathrm{~mol} \% \mathrm{JP}-10\right.$ and $8.72 \mathrm{~mol} \% \mathrm{O}_{2}$ in $\left.\mathrm{N}_{2}\right)$ in an isothermal batch reactor operated at $1000 \mathrm{~K}$ and $1.7 \mathrm{~atm}$. Rates are expressed relative to the total JP-10 consumption rate and are reported at a conversion of $10 \%$. The values for the pyrolysis are written in normal font while those for the stoichiometric combustion are indicated in italic and bold.

\section{Pyrolysis and oxidation shock tube speciation}

The shock tube results showing both experimental data points and model predictions are presented in Figure 6 to Figure 9. CHEMKIN-PRO ${ }^{37}$ was used to simulate the experimental shock tube conditions in an isothermal isobaric batch reactor with an initial loading of $0.2 \%$ mol JP-10 with oxygen at fuel-to-oxidation equivalence ratios of 1.0, 0.24, and 0.14 diluted in Ar. The main decomposition products were found to be ethene, cyclopentadiene, cyclopentene, propene, allene and aromatics such as benzene and toluene. Figure 6 shows that as expected, JP10 decomposes faster under leaner conditions. The main reason for this is that oxygen speeds up initiation of the radical mechanism by abstracting hydrogen atoms from JP-10 and also participates in chain branching ratios which increase the total number of radicals in the system. An important chain branching reaction in this regard is reaction ( $\mathrm{RXN}-4)$, in which one $\mathrm{H}$ radical reacts with $\mathrm{O}_{2}$ to form two reactive radicals: 


$$
\mathrm{H}+\mathrm{O}_{2} \rightarrow \mathrm{OH}+\mathrm{O}
$$

The model is able to predict the decomposition rate of JP-10 as a function of the temperature but tends to underpredict the yields of the major decomposition products (ethene and cyclopentadiene) by a factor of three. Propene and allene, on the other hand, are predicted fairly well by the model. In particular, the dependence of the propene yield on equivalence ratio is captured accurately for all studied equivalence ratios. Since most propene is formed through hydrogen abstractions by allyl from the parent molecule, the model proves to accurately capture the hydrogen abstractions from the parent fuel and manages to accurately describe the influence of oxygen on the radical pool, responsible for those hydrogen abstraction reactions. This is also supported by the good agreement for the JP-10 decomposition profiles, which are dominated by hydrogen abstraction reactions.

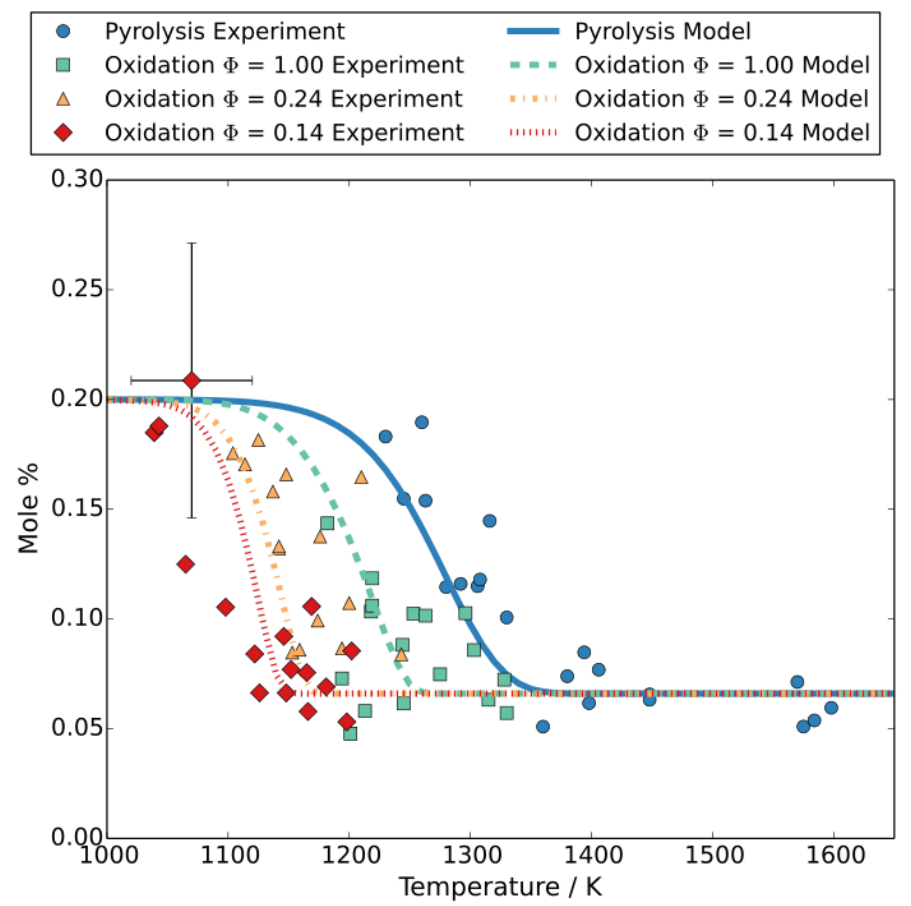

Figure 6. Experimentally measured (data points) and simulated concentration profiles (lines) of JP-10 as a function of temperature. Pyrolysis and oxidation experiments were conducted in a shock tube with an initial JP-10 loading of $0.2 \mathrm{~mol} \%$ diluted in Ar, $P=7 \mathrm{~atm}$, and $\tau=500 \mu \mathrm{s}$. Experimental error is shown on a single data point to illustrate uncertainties in concentration $( \pm 30 \%)$ and in temperature $( \pm 50 \mathrm{~K})$.

Figure 8 illustrates that the model accurately captures the formation of most aromatic compounds and the important intermediates leading to aromatic products, such as 1,3cyclohexadiene. Under pyrolysis conditions and a residence time of $500 \mu \mathrm{s}$, most aromatic components (indene, toluene, styrene...) have a maximum yield around 1400 to $1500 \mathrm{~K}$. The 1,3-cyclohexadiene and ethylbenzene concentrations peak at slightly lower temperatures as they are the main intermediates leading to benzene and styrene, respectively. Although most oxidation experiments were performed at lower temperatures $(1000-1200 \mathrm{~K})$ where pre-ignition products 
can be recovered, the experimental data suggest that the observed maximum in the yield curves for aromatics shifts to lower temperatures. This is also supported by the model. In Figure 9, the profiles of the main $\mathrm{C}_{3}$ and $\mathrm{C}_{4}$ species are shown. Once again, the model succeeds to reproduce most components within a factor of two to three. The largest discrepancies between the model and experiments are found for 1,3-butadiene, in particular for the leanest oxidation case $(\Phi=$ 0.14 ), and for butene. The model systemically underestimates the butene yield by a factor of 2 to 3 but seems to be able to accurately reproduce the temperature at which the butene yield will be maximal. 


\begin{tabular}{|llll|}
\hline & Pyrolysis Experiment & & Pyrolysis Model \\
$\square$ & Oxidation $\Phi=1.00$ Experiment & $==$ & Oxidation $\Phi=1.00$ Model \\
$\Delta$ & Oxidation $\Phi=0.24$ Experiment & $\ldots \cdots$ & Oxidation $\Phi=0.24$ Model \\
$\diamond$ & Oxidation $\Phi=0.14$ Experiment & $\ldots \ldots$ & Oxidation $\Phi=0.14$ Model \\
\hline
\end{tabular}
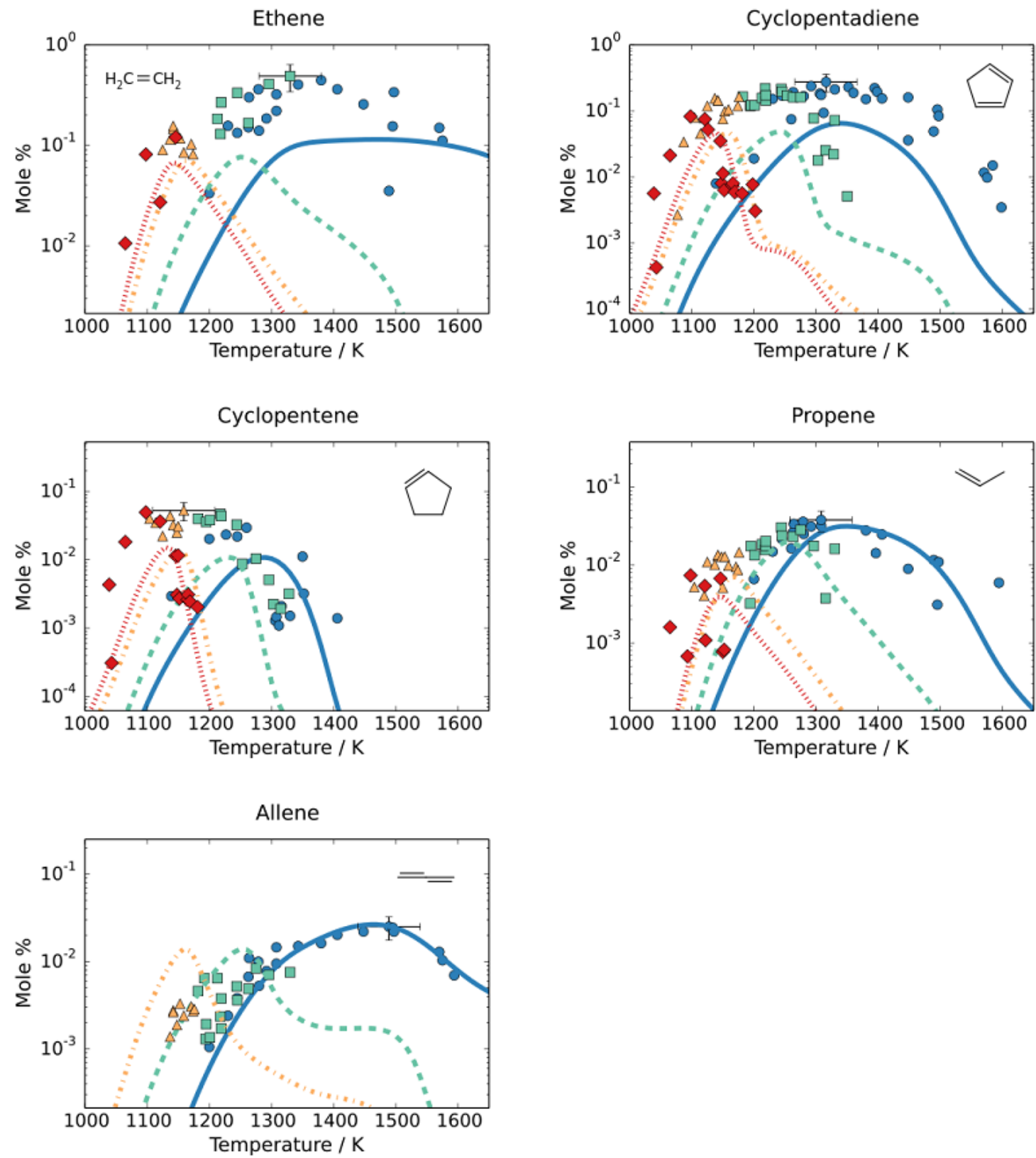

Figure 7. Experimentally measured (data points) and simulated concentration profiles (lines) of JP-10's main decomposition products as a function of temperature. Pyrolysis and oxidation experiments were conducted in a shock tube with an initial JP-10 loading of $0.2 \mathrm{~mol} \%$ diluted in $\mathrm{Ar}, P=7 \mathrm{~atm}$, and $\tau=500 \mu \mathrm{s}$. Experimental error bars are shown on a single data point to illustrate uncertainties in concentration $( \pm 30 \%)$ and in temperature $( \pm 50 \mathrm{~K})$. 

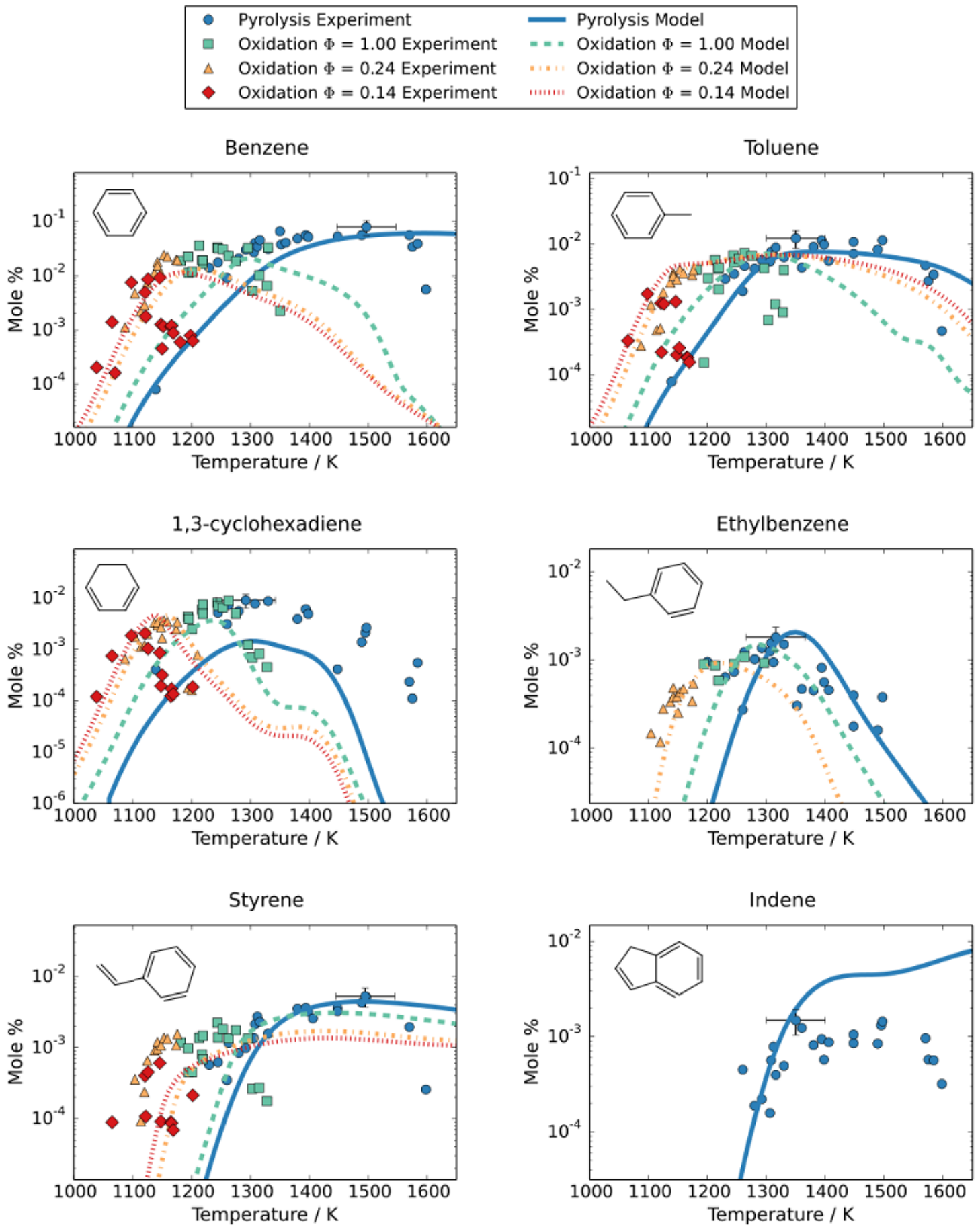

Figure 8. Experimentally measured (data points) and simulated concentration profiles (lines) of aromatics and related species from JP-10's decomposition as a function of temperature. Pyrolysis and oxidation experiments were conducted in a shock tube with an initial JP-10 loading of $0.2 \mathrm{~mol} \%$ diluted in Ar, $P=7 \mathrm{~atm}$, and $\tau=500 \mu \mathrm{s}$. Experimental error bars are shown on a single data point to illustrate uncertainties in concentration $( \pm 30 \%)$ and in temperature $( \pm 50 \mathrm{~K})$. 

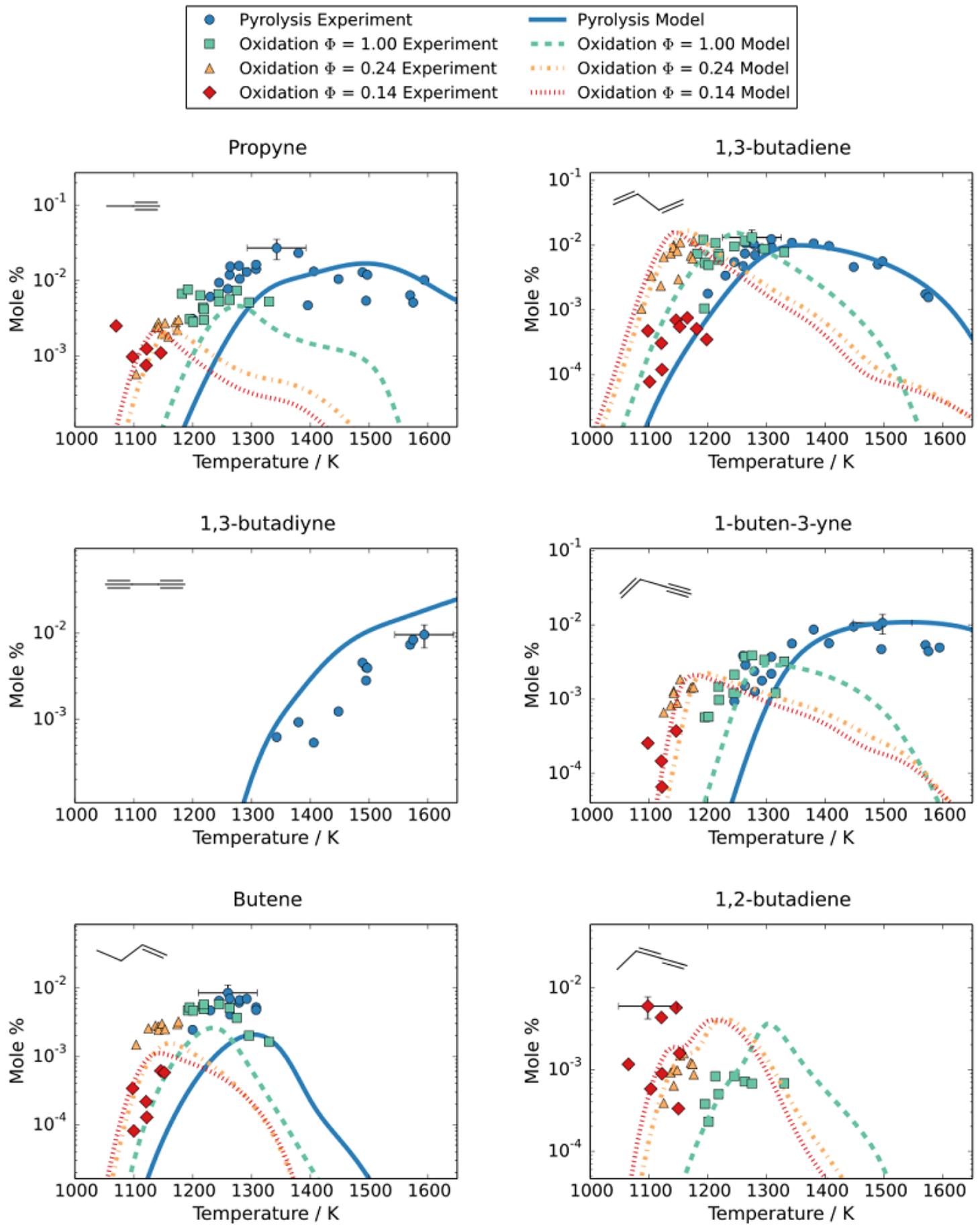

Figure 9. Experimentally measured (data points) and simulated concentration profiles (lines) of $\mathrm{C}_{3}$ and $\mathrm{C}_{4}$ species from JP-10's decomposition as a function of temperature. Pyrolysis and oxidation experiments were conducted in a shock tube with an initial JP-10 loading of $0.2 \mathrm{~mol} \%$ diluted in Ar, $P=7 \mathrm{~atm}$, and $\tau=$ $500 \mu \mathrm{s}$. Experimental error bars are shown on a single data point to illustrate uncertainties in concentration $( \pm 30 \%)$ and in temperature $( \pm 50 \mathrm{~K})$. 
Species that have been systematically observed during the pyrolysis experiments but are missing in the model are summarized in Table 2 (details can be found in the Supporting Information.) The main missing components are 1,3,5-cycloheptatriene and 2-propenylidene cyclobutene. Both products are structural isomers of toluene. We expect that 1,3,5cycloheptatriene and 2-propenylidene cyclobutene are predominantly formed by isomerization reactions on the $\mathrm{C}_{7} \mathrm{H}_{7}$ surface followed by hydrogen abstraction reactions. Other missing components are mostly (de)hydrogenated forms of some of the major reactions products. Phenylethyne is obtained by dehydrogenation of styrene while indane and 1,3-cycloheptadiene are the hydrogenated products of indene and 1,3,5-cycloheptatriene. The formation of 4-ethyl 3ethylidene cyclohexene and 1,2-divinyl cyclohexane further support the newly discovered reaction paths towards benzene. Intramolecular hydrogen shifts in the major intermediates, such as 4-ethylbicyclo[3.2.1]oct-2-ene (RXN-5), can easily lead to the observed species.

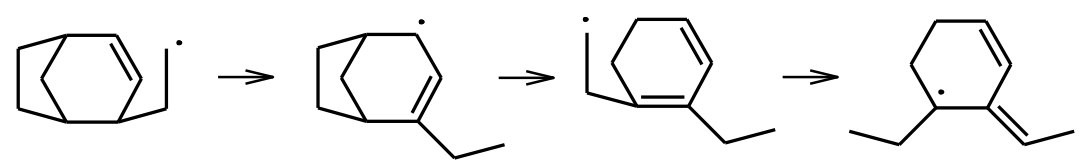

$(\mathrm{RXN}-5)$

Table 2. Species that have been experimentally observed* but are missing in the model. The peak concentrations listed are from the shock tube pyrolysis experiments. The initial JP-10 feed was 0.2 mol\%.

\begin{tabular}{cccc}
\hline Name & $\begin{array}{c}\text { Chemical } \\
\text { Formula }\end{array}$ & $\begin{array}{c}\text { Peak concentration (mol } \\
\% \text { ) }\end{array}$ \\
2-propenylidene cyclobutene & $\mathrm{C}_{7} \mathrm{H}_{8}$ & Structure \\
1,3,5-cycloheptatriene & $\mathrm{C}_{7} \mathrm{H}_{8}$ & $1.2 \times 10^{-2}$ \\
1,3-cycloheptadiene & $\mathrm{C}_{7} \mathrm{H}_{10}$ & \\
bicyclo(4.1.0)hept-2-ene & $\mathrm{C}_{7} \mathrm{H}_{10}$ & \\
\hline
\end{tabular}




$\begin{array}{ccc}\text { naphthalene } & \\ \begin{array}{c}\text { indane } \\ \text { 4-ethyl 3-ethylidene } \\ \text { cyclohexene } \\ \text { 1,2-divinyl cyclohexane }\end{array} & \mathrm{C}_{10} \mathrm{H}_{16} & \mathrm{C}_{10} \mathrm{H}_{16}\end{array}$

* Best guesses based on the mass spectrum and retention time

\section{Pyrolysis flow tube speciation}

A second validation for the model compares our predictions with the JP-10 pyrolysis flow tube experimental data reported by Vandewiele et al. ${ }^{34}$ These experiments were obtained in a bench scale setup recently used for studies on butanol and aromatic hydrocarbons. The experimental setup is extensively described in the previous papers ${ }^{38}$ and described briefly here. The reactor is a 1.475-m long, 6-mm internal diameter tube made of Incoloy $800 \mathrm{HT}(\mathrm{Ni}, 30-35$; $\mathrm{Cr}, 19$ - 23; and $\mathrm{Fe}>39.5 \mathrm{wt} \%$ ). The analysis section consists of a $\mathrm{GC} \times \mathrm{GC}$ equipped with a mass spectrometer to analyze the $\mathrm{C}_{5}+$ fraction, while the $\mathrm{C}_{4}$ - fraction is analyzed by a Refinery Gas Analyzer (RGA) equipped with two TCD's and a FID. Residence times varied around $0.4 \mathrm{~s}$ while conversions between 4 and $94 \%$ were obtained.

Two dilutions (7 and $10 \mathrm{~mol} \%$ ) of JP-10 in nitrogen were studied and the results are presented in comparison with model predictions in Figure 10 and Figure 11, with time traces computed as normalized mole percentages excluding nitrogen. It can be seen that the model is able to accurately reproduce the decomposition rate of JP-10 for both dilution conditions. At higher temperatures 1020 to $1080 \mathrm{~K}$, the model tends to overestimate the conversion of JP-10 by $20 \%$ and consequently slightly over predicts the yields of the main decomposition products.

The largest discrepancy between the model and simulated data is the methane yield. Experimentally it is observed that up to $15 \%$ of the products consist of methane (mole based), while the model only predicts values up to $10 \%$. This is directly related to a too low methyl concentration in the simulated radical pool, as methane is predominantly formed by hydrogen abstraction reactions involving the methyl radical. This discrepancy can have various origins, i.e. missing pathways that produce methyl or recombination/addition reactions involving the methyl radical that are too fast. Due to the overestimation of the toluene concentration, we suspect that part of the underestimation of the methyl concentration is due to missing either some of the 
addition reactions of hydrogen radicals to methylated aromatics (toluene, xylene...) or missing some of the elimination reactions leading to $\mathrm{CH}_{3}$ radicals, e.g. (RXN-6). Due to breaking aromaticity in the ring, some of the independent addition reactions may be too slow to be included by RMG, however, the sum of their contributions can be significant.

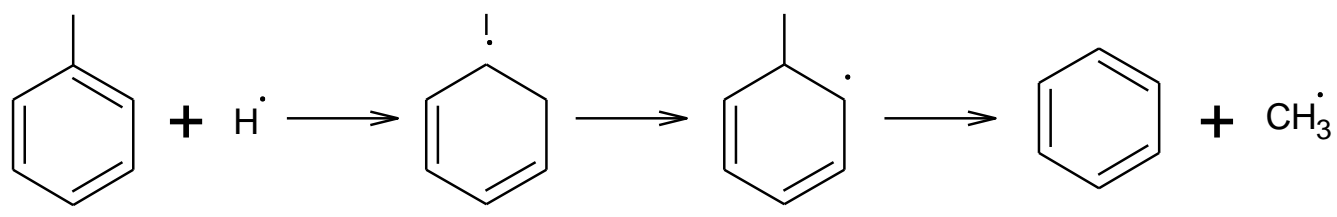

(RXN-6)

Figure 10 and Figure 11 illustrate that temperature dependencies and relative yields for the major components are reproduced well by the model. Both the experimental data and model predict a maximum for propene and cyclopentadiene at temperatures around $1050 \mathrm{~K}$. Most aromatic and minor components are reproduced within a factor of two, again capturing most temperature dependencies. Trace components such as ethyne, propyne and allene are also predicted by the model and generally agree within a factor of 4 with the experimental data. Contradicting the model is the higher concentration 10 mol\% JP-10 pyrolysis experiment showing a decreasing propyne yield with increasing temperature. However, this trend is reversed in the $7 \mathrm{~mol} \%$ experiment, where the propyne yield increases with temperature as predicted by the model.

An important missing species in the model is naphthalene, which accounts for approximately $1 \mathrm{~mol} \%$ of the measured product distribution excluding $\mathrm{N}_{2}$ at a temperature of $1080 \mathrm{~K}$ in the experiments. We found that in the lower temperature region (1000 to $1100 \mathrm{~K}$ ), the rate coefficient reported by Murakami et al. ${ }^{39}$ for the reaction:

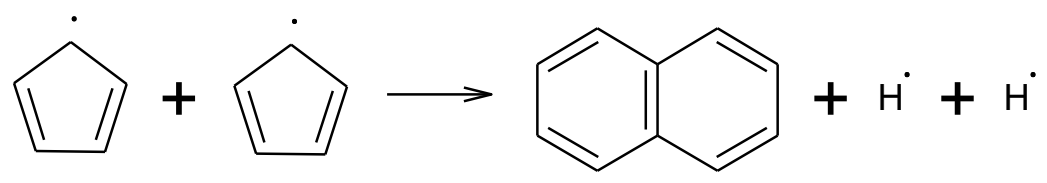

(RXN-7)

is able to predict the observed naphthalene quite well. However, we opted not to include this reaction in the network since at higher temperatures (1300 to $1400 \mathrm{~K})$, this rate leads to an over prediction of the naphthalene yield by a factor of 5 . This systematic over prediction of the naphthalene yield by inclusion of (RXN-7) using the Murakami et al. rate coefficient has also been observed by other authors. ${ }^{40}$ 

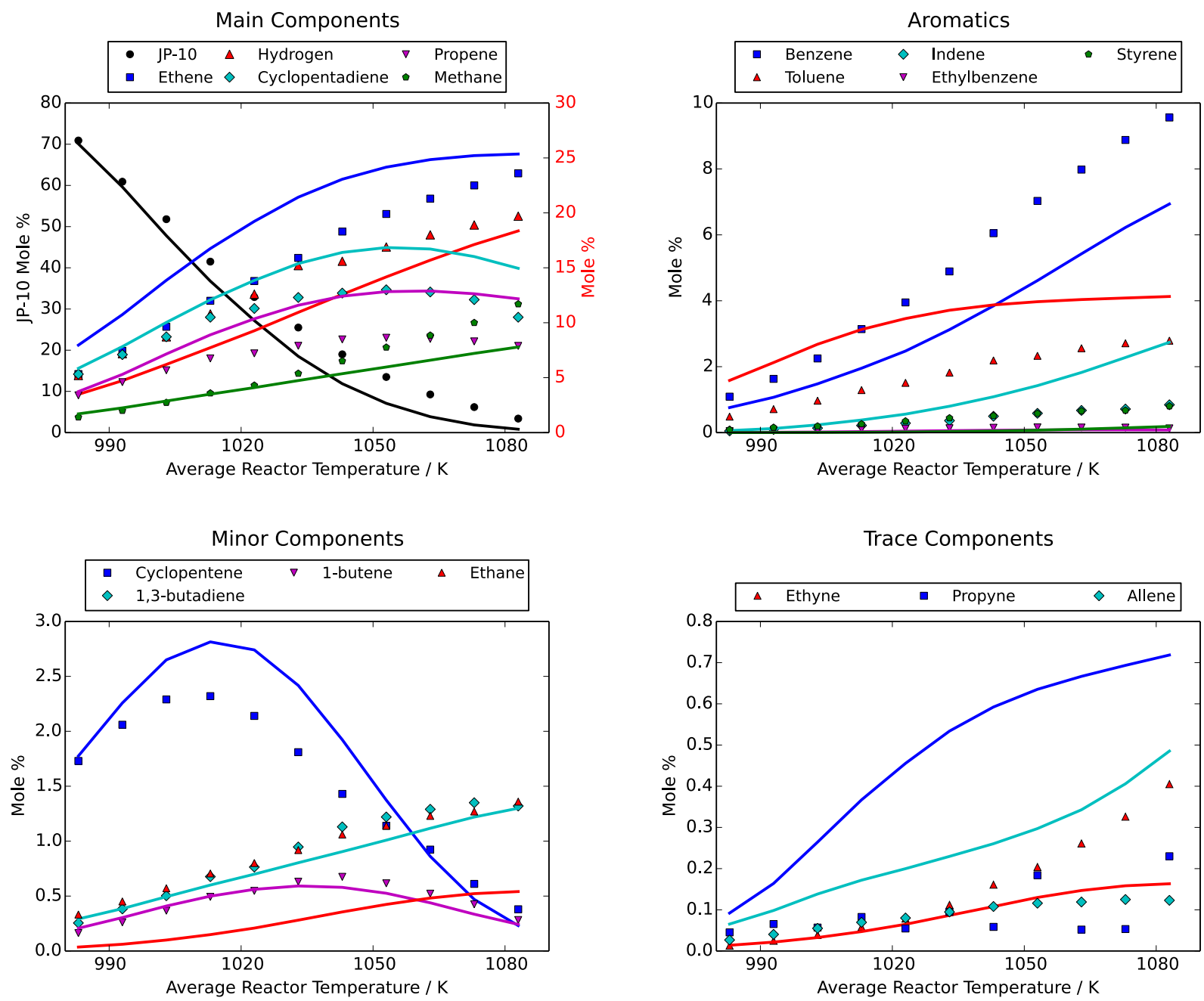

Figure 10. Experimentally measured (data points) and simulated concentration profiles (lines) as a function of temperature for the pyrolysis of JP-10 as reported by Vandewiele et al. ${ }^{34}$ Experimental conditions: $7 \mathrm{~mol} \% \mathrm{JP}-10$ diluted in $\mathrm{N}_{2}, \mathrm{P}=\mathbf{0 . 1 4} \mathrm{MPa}$, mass flow rate of $\mathrm{JP}-10=2.3310^{-2} \mathrm{~g} \mathrm{~s}^{-1}$. Mole percentages are normalized excluding $\mathbf{N}_{2}$. 

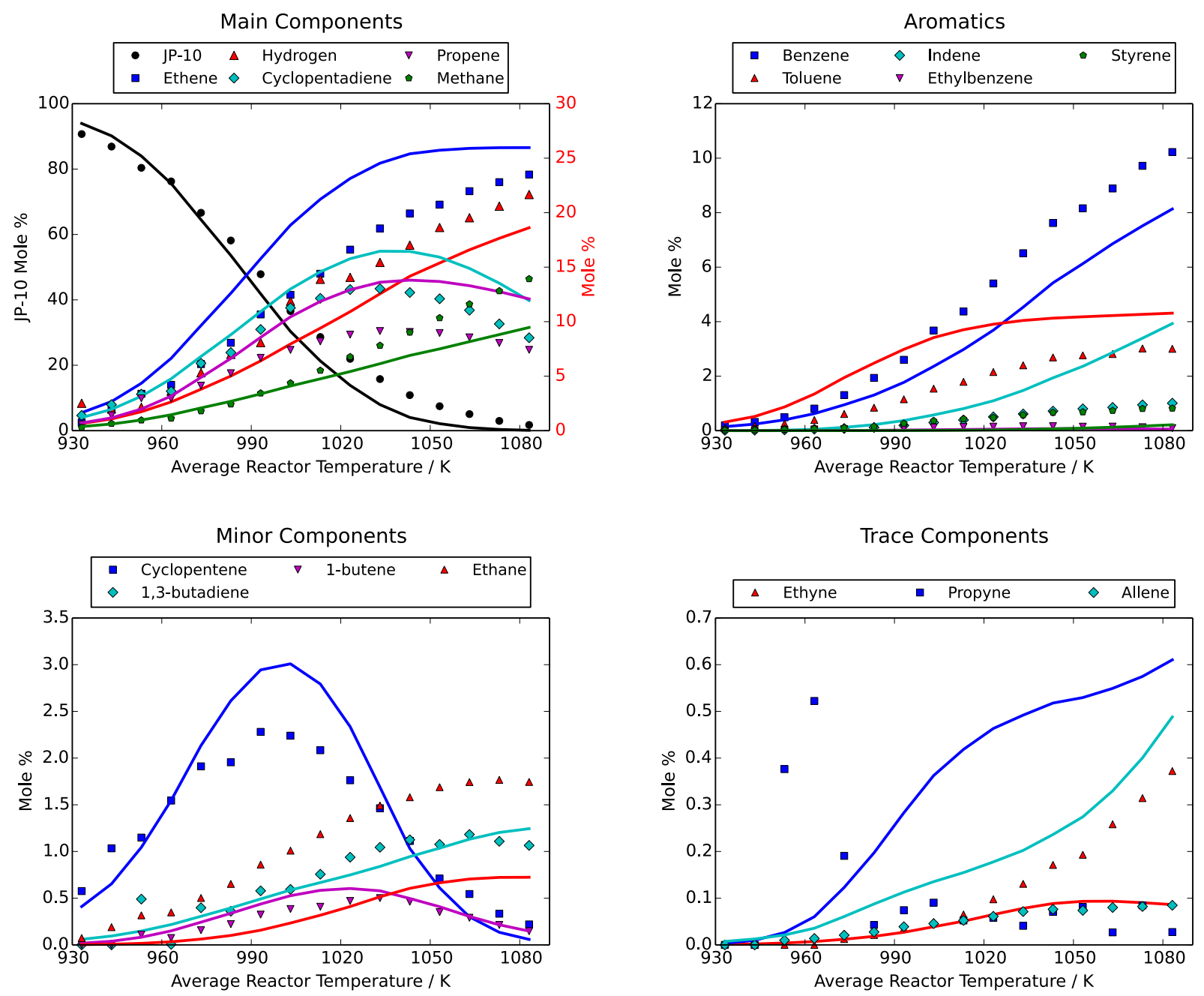

Figure 11. Experimentally measured (data points) and simulated concentration profiles (lines) as a function of temperature for the pyrolysis of JP-10 as reported by Vandewiele et al. ${ }^{34}$ Experimental conditions: $10 \mathrm{~mol} \% \mathrm{JP}-10$ diluted in $\mathrm{N}_{2}, \mathrm{P}=\mathbf{0 . 1 4} \mathrm{MPa}$, mass flow rate of $\mathrm{JP}-10=2.3310^{-2} \mathrm{~g} \mathrm{~s}^{-1}$. Mole percentages are normalized excluding $\mathbf{N}_{2}$.

\section{Ignition delays}

Four experimental datasets for JP-10 ignition delay can be found in literature. ${ }^{7-10}$ The summary of the experimental conditions and ignition delay measurement metrics can be found in Table 3. The expression $\tau=\mathrm{A} \cdot \mathrm{P}^{\mathrm{n}} \cdot \exp (\mathrm{B} / \mathrm{T})$ can be used to correlate the experimental data (where $\mathrm{P}$ is in atmospheres and $\mathrm{T}$ in Kelvin). By fitting a unique correlation for each dataset at the given mole $\% \mathrm{JP}-10$ and stoichiometric ratio, pressure corrected ignition delays $\tau_{\mathrm{P}}=\left(\mathrm{P} / \mathrm{P}_{0}\right)^{\mathrm{n}} \tau_{0}$ can be derived, allowing datasets amongst multiple pressures to be compared. The fitted parameters for these correlations can be found in the Supporting Information. In Figure 12, the stoichiometric ignition delays from all four datasets are pressure-corrected to $\mathrm{P}_{0}=7$ atm and compared with simulated ignition delays. It can be seen from this figure that there are some discrepancies amongst the existing datasets. Though the Colket and Spadaccini ${ }^{8}$ experiments 
were conducted at lower feed concentration ( 0.15 mol\% JP-10), the ignition delays were comparatively fast compared to the Davidson et al. ${ }^{7}$ experiments conducted at slightly higher feed concentrations (0.20 mol\% JP-10). The Mikolaitis et al. dataset has the fastest ignition delays, as expected from a higher feed concentration (1.48 mol\% JP-10). The apparent activation energy of ignition (the slope of the correlation) for the Wang et al. ${ }^{10}$ ignition delay data appears to be smaller than the for the other datasets. The discrepancies between these datasets may be due to slight differences in the ignition delay metrics employed, as well as accuracy of the JP-10 concentration measurements. The Davidson and Mikolaitis datasets used the peak $\mathrm{CH}^{*}$ emission as the indicator of ignition, while both Colket and Wang experiments used initial rise onsets of the $\mathrm{CH}^{*}$ emission, leading to faster measured ignition times. Mikolaitis and coworkers ${ }^{9}$ found that at intermediate temperatures, two emission peaks for ignition delay were present. The first peak ignition delays from the $\mathrm{CH}$ emission were recommended by the authors the true indicator of ignition and are compared with the model predictions here.

Table 3. Summary of experimental conditions for ignition delay datasets available in literature.

\begin{tabular}{|c|c|c|c|c|c|}
\hline Dataset & $\begin{array}{c}\text { Temperature } \\
\text { range }\end{array}$ & $\begin{array}{c}\text { Pressure } \\
\text { range }\end{array}$ & $\begin{array}{c}\text { Equivalence } \\
\text { ratios }(\Phi)\end{array}$ & $\begin{array}{c}\text { Bath } \\
\text { gas }\end{array}$ & $\begin{array}{c}\text { Ignition delay } \\
\text { metric }\end{array}$ \\
\hline Colket and Spadaccini ${ }^{8}$ & $1250-1500 \mathrm{~K}$ & 3-7 atm & $0.5-1.5$ & $\mathrm{Ar}$ & $\begin{array}{c}\text { Initial rise onset in } \\
\mathrm{OH}^{*} \text { emission }\end{array}$ \\
\hline Davidson et al. ${ }^{7}$ & $1300-1700 \mathrm{~K}$ & $1-6 \mathrm{~atm}$ & $0.5-2.0$ & $\mathrm{Ar}$ & $\begin{array}{c}\text { Peak CH* } \\
\text { emission }\end{array}$ \\
\hline Mikolaitis et al. ${ }^{9}$ & $1200-2000 \mathrm{~K}$ & $10-25 \mathrm{~atm}$ & 1.0 & $\mathrm{~N}_{2}$ & $\begin{array}{c}\text { Peak CH* } \\
\text { emission }\end{array}$ \\
\hline Wang et al. ${ }^{10}$ & $1000-2100 \mathrm{~K}$ & $1.5-5.5 \mathrm{~atm}$ & $0.25-2.0$ & $\mathrm{Ar}$ & $\begin{array}{l}\text { Initial rise onset in } \\
\mathrm{CH}^{*} \text { emission for } \\
\qquad \Phi=1.0 \\
\text { experiments, } \\
\text { initial rise onset in } \\
\mathrm{OH}^{*} \text { emission for } \\
\text { all other } \\
\text { experiments }\end{array}$ \\
\hline
\end{tabular}



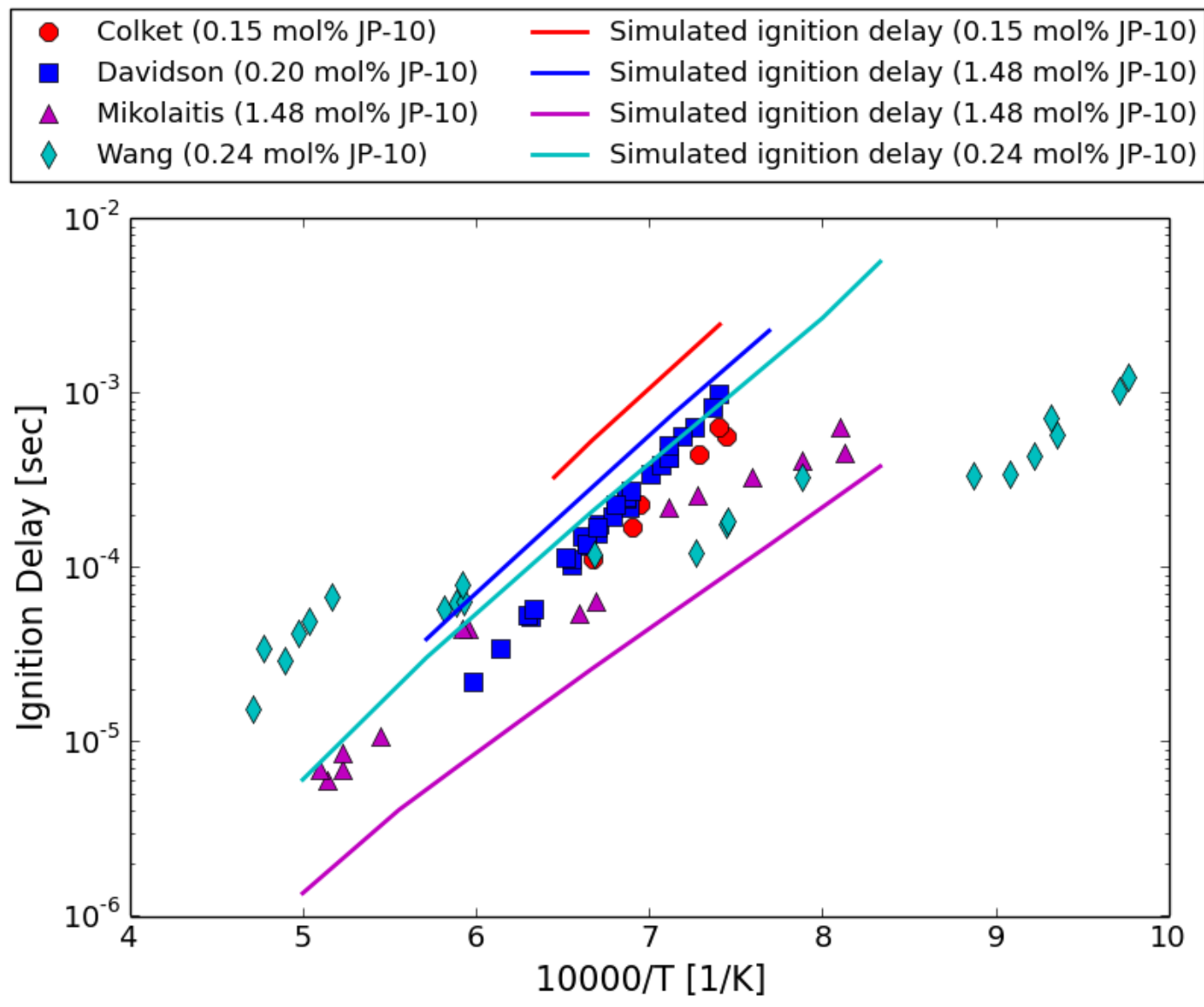

Figure 12. Pressure corrected (7 atm) experimental ignition delays for Colket (0.15 mol\% JP-10 feed, circles), Davidson (0.20 mol\% JP-10 feed, squares), Wang (0.24 mol\% JP-10 feed, diamonds), and Mikolaitis (1.48 mol\% JP-10 feed, triangles) at stoichiometric conditions, along with simulated ignition delays (lines) at $\Phi=1.0$ and $P=7$ atm .

Ignition delays at stoichiometric conditions were simulated using the model at $7 \mathrm{~atm}$ and experimental feed conditions and compared with experiment in Figure 12. The maximum in the $\left[\mathrm{C}_{2} \mathrm{H}\right][\mathrm{O}]$ product was used as the metric for ignition delay in the simulations. Since $\mathrm{CH}^{*}$ emissions occur primarily via the reaction $\mathrm{C}_{2} \mathrm{H}+\mathrm{O} \rightarrow \mathrm{CH}^{*}+\mathrm{CO}$, the $\left[\mathrm{C}_{2} \mathrm{H}\right][\mathrm{O}]$ product most effectively simulates experimental $\mathrm{CH}$ emissions used to measure ignition delay. ${ }^{7}$ In Figure 12, we see that the simulated ignition delays are slower than experiment for the Davidson and Colket datasets, but systematically faster than the Mikolaitis ignition delays. However, the predicted apparent activation energy for ignition agrees well with all datasets except the Wang experiments.

In Figure 13, a parity plot is shown between experimentally observed and modeled ignition delay times for all four experimental datasets over all experimental conditions. It can be seen from Figure 13 that the model succeeds to reproduce all reported ignition delays covering a broad range of temperatures to mostly within a factor of 4 . 
In particular, the experimental ignition delays measured by Davidson and by Colket are mostly reproduced by the model (within a factor of 2 to 3 ).

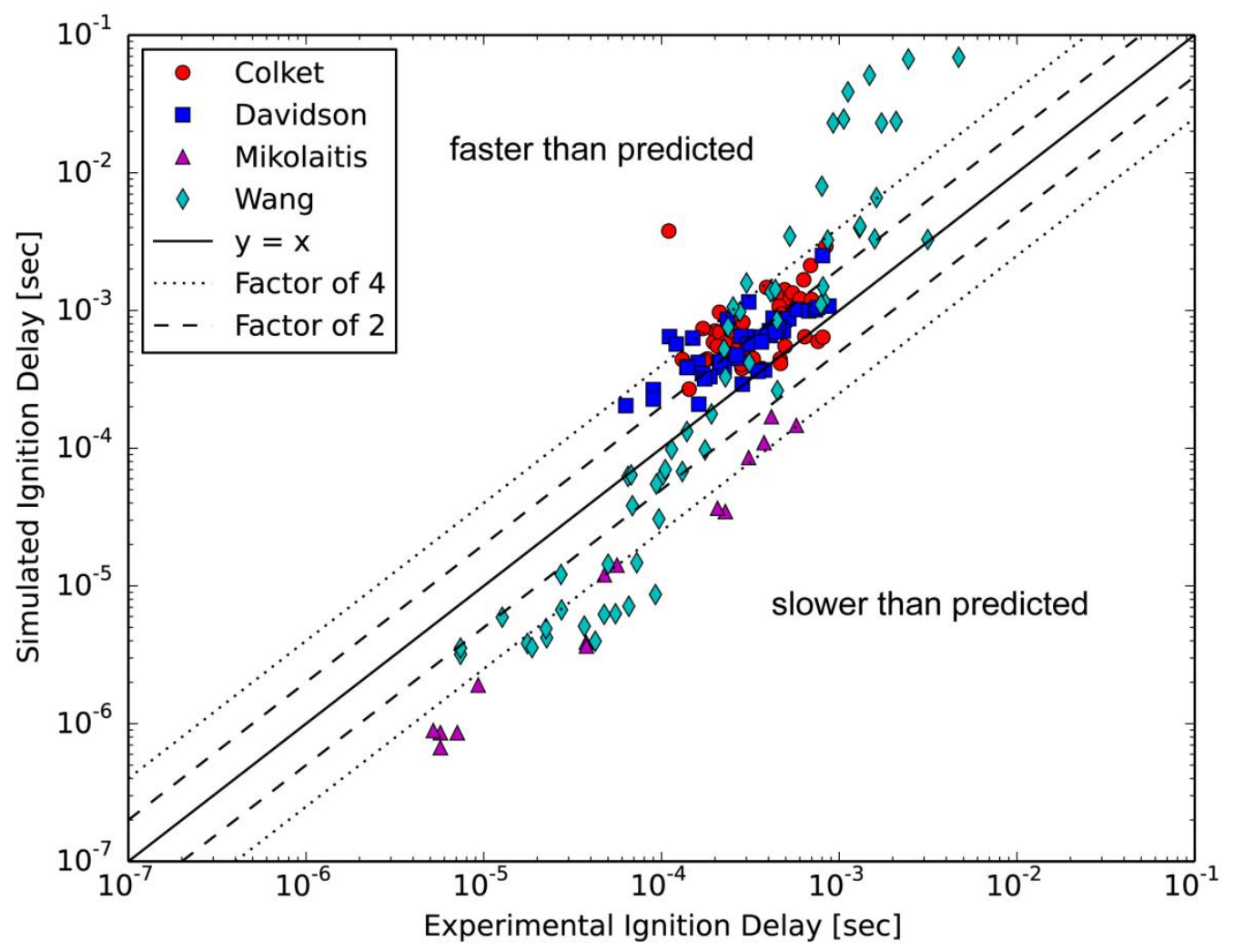

Figure 13. Parity plot comparing simulated and measured ignition time delays for JP-10 for existing experimental datasets: Colket (circles); Davidson (squares); Mikolaitis (triangles); and Wang (diamonds).

$\mathrm{OH}$ sensitivity analysis was performed for the model at stoichiometric conditions $(1500 \mathrm{~K}$ and 7 atm for a 0.20 mol\% feed JP-10 in argon, 50\% JP-10 conversion), with the most important reactions presented in Figure 14. In agreement with Davidson et al. $^{2}$, we find that the $\mathrm{OH}$ concentration is most sensitive to the branching reaction $\mathrm{H}+\mathrm{O}_{2} \rightarrow \mathrm{O}+\mathrm{OH}$. Davidson's model used a modified version of the Lindstedt and Maurice mechanism ${ }^{41}$ and found that the $\mathrm{OH}$ concentration is primarily sensitive to $\mathrm{C}_{2}$ oxidation reactions. Figure 14 illustrates that besides the $\mathrm{C}_{2}$ oxidation chemistry, ignition delays are also sensitive to reactions with some of the decomposition products. These reactions are mainly recombination reactions or hydrogen abstraction reactions involving long-lived, resonantly stabilized radicals such as cyclopentadienyl and allyl. The largest negative sensitivity coefficient is obtained for the recombination of $\mathrm{H}$ with allyl forming propene, followed by the recombination of $\mathrm{H}$ with cyclopentadienyl forming cyclopentadiene and the recombination of $\mathrm{H}$ with propargyl radical to produce allene. Particularly in oxidation conditions, resonantly stabilized radicals can accumulate in larger concentrations and combine with the relatively unreactive $\mathrm{HO}_{2}$ radical, 
contributing to chain branching reactions that accelerate ignition. ${ }^{42}$ For instance, the sensitive reaction of cyclopentadienyl radical with $\mathrm{HO}_{2}$ can lead to active $\mathrm{OH}$ radical generation through the dissociation of the $\mathrm{RO}-\mathrm{OH}$ bond in the hydroperoxide product. This shows that a predictive model for ignition delays requires (i) correct small molecule chemistry and (ii) a correct treatment of the main stable radicals and their interaction with the radical pool.

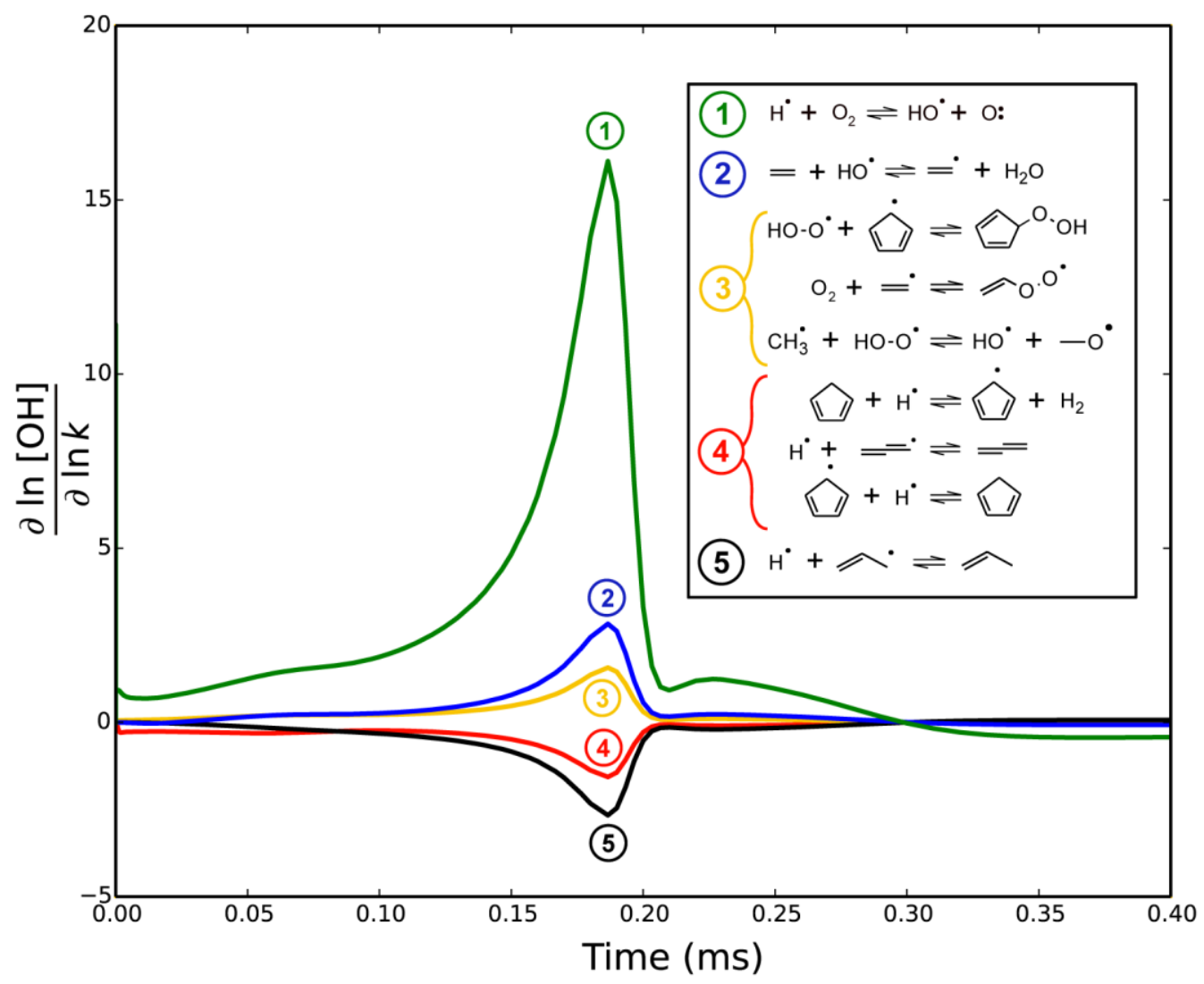

Figure 14. Most sensitive reaction kinetics found in normalized $\mathrm{OH}$ sensitivity analysis $(\mathrm{d} \ln [\mathrm{OH}] / \mathrm{dln} \mathrm{k})$ conducted at $1500 \mathrm{~K}, 7 \mathrm{~atm}, \Phi=1.0,0.20 \mathrm{~mol} \% \mathrm{JP}-10$ diluted in Ar in an adiabatic batch reactor. Reactions grouped together have nearly equal importance.

\section{Conclusions}

Despite JP-10's popularity as a jet propellant, few detailed kinetics models for JP-10 combustion exist, and most are unvalidated against pre-ignition product distributions, mainly due to lack of experimental data. This work aims to gain new insights on JP-10's complex decomposition chemistry through a combination of experimental and modeling efforts. Experiments were carried out in single-pulse shock tubes at pyrolysis and oxidation conditions, ranging from very lean $(\Phi=0.14)$ to stoichiometric $(\Phi=1.0)$, with products analyzed using 
GC/MS. The experiments show that JP-10 decomposes primarily to ethene, propene, cyclopentadiene, and aromatics consisting mostly of benzene and toluene. GC/MS allowed for the identification of a variety of trace components such as 1,2-divinylcyclohexane, butadiene and 1,3-cyclohexadiene. Though minor, these components are relatively important from a modeling viewpoint as they help to elucidate intermediate pathways in JP-10's decomposition mechanism.

The kinetic model was developed using RMG, an automatic reaction mechanism generator which uses a rate based termination criteria to include species with the most significant fluxes to a reaction network. Four models were generated, covering temperatures from $1000 \mathrm{~K}$ up to $2000 \mathrm{~K}$ with equivalence ratios ranging from very lean $(\Phi=0.14)$ to pyrolysis conditions. The final merged network contains almost 700 species reacting through more than 15000 reactions. Despite many similarities with existing mechanisms, this is the first model that contains multiple reaction paths from the parent fuel towards benzene. At lower temperatures $(<1000 \mathrm{~K})$, benzene formation involves the formation of a tricyclo[4.2.1.1(2,5)]decyl radical that undergo four successive $\beta$-scissions to form cyclohexadienyl and two ethene molecules. Higher temperatures favor an alternate reaction path that involves the formation of a bicyclo(3.1.0)hexane ring structure leading to butadiene and cyclohexenyl. These reaction paths are supported by the identification of intermediate species by experiment and also backed up by theory.

The generated model was thoroughly validated against the new experimental data and literature data and succeeds in accurately simulating JP-10 conversion rates under all equivalence ratios. The model captures most temperature dependencies in the shock tube product distributions, with agreement between the modeled and experimentally observed product concentrations generally within a factor of 3 . The model was also validated using the speciation data from recent pyrolysis experiments conducted by the Ghent group, succeeding in predicting the major products, ethene and cyclopentadiene, within $30 \%$ and reproducing most other product concentrations within a factor of 2. Experimentally observed ignition delays over four existing literature datasets are reproduced by the model mostly within a factor of 4 . Sensitivity analyses show that small molecule chemistry is controlling for the ignition delays at high temperature. We conclude that the combined efforts of experimentalists, theoreticians and modelers has led to a new JP-10 model that is able to reliably reproduce speciation data and ignition delays over a wide range of temperatures and equivalence ratios, as well as elucidate new pathways towards aromatics formation.

\section{Acknowledgements}

The authors gratefully acknowledge financial support from the Naval Air Warfare Center under contracts N68335-09-C-0367 and N68335-10-C-0534, as well as program support by Mr. Richard Burnes. We also gratefully acknowledge funding for RMG development by the Department of Energy, Office of Basic Energy Sciences through grant DE-FG02-98ER14914 and by the National Science Foundation under grants No. 0312359 and 0535604. 
Aäron Vandeputte acknowledges the financial support of the Combustion Energy Frontier Research Center, funded by the U.S. Department of Energy, Office of Basic Energy Sciences under award number DE-SC0001198.

Dr. Wing Tsang of NIST offered helpful suggestions in the early phase of the shock tube experiments, which are gratefully acknowledged.

\section{Supporting information}

A CHEMKIN-format version of the present model, rate coefficients obtained via quantum chemistry calculations, and additional experimental shock tube results are included in the Supporting Information.

\section{References}

1. (a) Bruno, T. J.; Huber, M. L.; Laesecke, A.; Lemmon, E. W.; Perkins, R. A., Thermochemical and thermophysical properties of JP-10. 2006, NISTIR 6640; (b) Chung, H. S.; Chen, C. S. H.; Kremer, R. A.; Boulton, J. R.; Burdette, G. W., Recent developments in highenergy density liquid hydrocarbon fuels. Energy \& Fuels 1999, 13 (3), 641-649; (c) Joppin, C. Cooling Performance of Storable Propellants for a micro rocket engine. Massachusetts Institute of Technology, Cambridge, MA, 2002.

2. Davidson, D. F.; Horning, D. C.; Oehlschlaeger, M. A.; Hanson, R. K., The Decomposition Products of JP-10. AIAA 01-3707 37th Joint Propulsion Conference 2001.

3. Nakra, S.; Green, R. J.; Anderson, S. L., Thermal decomposition of JP-10 studied by micro-flowtube pyrolysis-mass spectrometry. Combustion and Flame 2006, 144 (4), 662-674.

4. Striebich, R. C.; Lawrence, J., Thermal decomposition of high-energy density materials at high pressure and temperature. Journal of Analytical and Applied Pyrolysis 2003, 70 (2), 339352.

5. Xing, Y.; Yang, X.; Fang, W. J.; Guo, Y. S.; Lin, R. S., Measurement on vapor pressure, density and viscosity for binary mixtures of JP-10 and methylcyclohexane. Fluid Phase Equilibria 2011, 305 (2), 192-196.

6. Herbinet, O.; Sirjean, B.; Bounaceur, R.; Fournet, R.; Battin-Leclerc, F.; Scacchi, G.; Marquaire, P. M., Primary mechanism of the thermal decomposition of tricyclodecane. Journal of Physical Chemistry A 2006, 110 (39), 11298-11314.

7. Davidson, D. F.; Horning, D. C.; Herbon, J. T.; Hanson, R. K., Shock tube measurements of JP-10 ignition. Proceedings of the Combustion Institute 2000, 28, 1687-1692.

8. Colket, M. B.; Spadaccini, L. J., Scramjet fuels autoignition study. Journal of Propulsion and Power 2001, 17 (2), 315-323.

9. Mikolaitis, D. W.; Segal, C.; Chandy, A., Ignition delay for jet propellant 10/air and jet propellant 10/high-energy density fuel/air mixtures. Journal of Propulsion and Power 2003, 19 (4), 601-606.

10. Wang, S.; Gou, H. J.; Fan, B. C.; He, Y. Z.; Zhang, S. T.; Cui, J. P., Shock tube study of JP-10 ignition delay time. Chinese Journal of Chemical Physics 2007, 20 (1), 48-52. 
11. Zehe, M. J.; Jaffe, R. L., Theoretical Calculation of Jet Fuel Thermochemistry. 1. Tetrahydrodicylopentadiene (JP10) Thermochemistry Using the CBS-QB3 and G3(MP2)//B3LYP Methods. The Journal of Organic Chemistry 2010, 75 (13), 4387-4391.

12. Bozzelli, J. W.; Castillo, A.; Hudzik, J. M., Exo-tricyclo[5.2.1.0(2,6)]decane (TCD or JP10) decomposition: Thermochemostry and kinetics of ring opening and formed intermediates. Abstracts of Papers of the American Chemical Society 2012, 244.

13. Chenoweth, K.; van Duin, A. C. T.; Dasgupta, S.; Goddard, W. A., Initiation Mechanisms and Kinetics of Pyrolysis and Combustion of JP-10 Hydrocarbon Jet Fuel. Journal of Physical Chemistry A 2009, 113 (9), 1740-1746.

14. Yue, L.; Xie, H. J.; Qin, X. M.; Lu, X. X.; Fang, W. J., A DFT study on the thermal cracking of JP-10. Journal of Molecular Modeling 2013, 19 (12), 5355-5365.

15. Magoon, G. R.; Aguilera-Iparraguirre, J.; Green, W. H.; Lutz, J. J.; Piecuch, P.; Wong, H. W.; Oluwole, O. O., Detailed chemical kinetic modeling of JP-10 (exotetrahydrodicyclopentadiene) high-temperature oxidation: Exploring the role of biradical species in initial decomposition steps. International Journal of Chemical Kinetics 2012, 44 (3), 179-193.

16. Li, S. C.; Varatharajan, B.; Williams, F. A., Chemistry of JP-10 ignition. Aiaa Journal 2001, 39 (12), 2351-2356.

17. Green, W. H.; Allen, J. W.; Buesser, B. A.; Ashcraft, R. W.; Beran, G. J.; Class, C. A.; Gao, C.; Goldsmith, C. F.; Harper, M. R.; Jalan, A.; Keceli, M.; Magoon, G. R.; Matheu, D. M.; Merchant, S. S.; Mo, J. D.; Petway, S.; Raman, S.; Sharma, S.; Song, J.; Suleymanov, Y.; Van Geem, K. M.; Wen, J.; West, R. H.; Wong, A.; Wong, H. S.; Yelvington, P. E.; Yee, N.; Yu, J. RMG - Reaction Mechanism Generator v4.0.1 2013. http://rmg.sourceforge.net/

18. Vandewiele, N. M.; Magoon, G. R.; Van Geem, K. M.; Reyniers, M.-F.; Green, W. H.; Marin, G. B., Kinetic Modeling of Jet Propellant-10 Pyrolysis. Energy \& Fuels 2015, 29 (1), 413-427.

19. Lifshitz, A.; Bauer, S. H.; Resler, E. L., Studies with a Single-Pulse Shock Tube .1. CisTrans Isomerization of Butene-2. Journal of Chemical Physics 1963, 38 (9), 2056-2063.

20. (a) Green Jr, W. H.; Guy, B. M., Predictive Kinetics: A New Approach for the 21st Century. In Advances in Chemical Engineering, Academic Press: 2007; Vol. Volume 32, pp 150, 313; (b) Song, J. Building Robust Chemical Reaction Mechanisms: Next Generation of Automatic Model Construction. Doctoral, Massachusetts Institute of Technology, Cambridge, 2004; (c) Susnow, R. G.; Dean, A. M.; Green, W. H.; Peczak, P.; Broadbelt, L. J., Rate-based construction of kinetic models for complex systems. Journal of Physical Chemistry A 1997, 101 (20), 3731-3740.

21. Smith, G.; Wang, H., Personal Communication, 2014.

22. Ochterski, J. W.; Petersson, G. A.; Montgomery, J. A., A complete basis set model chemistry .5. Extensions to six or more heavy atoms. Journal of Chemical Physics 1996, 104 (7), 2598-2619.

23. Boese, A. D.; Martin, J. M. L., Development of density functionals for thermochemical kinetics. J Chem Phys 2004, 121 (8), 3405-3416.

24. Sharma, S.; Raman, S.; Green, W. H., Intramolecular Hydrogen Migration in Alkylperoxy and Hydroperoxyalkylperoxy Radicals: Accurate Treatment of Hindered Rotors. The Journal of Physical Chemistry A 2010, 114 (18), 5689-5701.

25. (a) Sabbe, M. K.; Reyniers, M.-F.; Van Speybroeck, V.; Waroquier, M.; Marin, G. B., Carbon-Centered Radical Addition and $\beta$-Scission Reactions: Modeling of Activation Energies and Pre-exponential Factors. ChemPhysChem 2008, 9 (1), 124-140; (b) Sabbe, M. K.; 
Vandeputte, A. G.; Reyniers, M.-F.; Waroquier, M.; Marin, G. B., Modeling the influence of resonance stabilization on the kinetics of hydrogen abstractions. Physical Chemistry Chemical Physics 2010, 12 (6), 1278-1298.

26. Magoon, G. R.; Green, W. H., Design and implementation of a next-generation software interface for on-the-fly quantum and force field calculations in automated reaction mechanism generation. Computers \& Chemical Engineering 2013, 52 (0), 35-45.

27. Stewart, J. J. P. MOPAC2012, Stewart Computational Chemistry: 2012. http://openmopac.net

28. Landrum, G. RDKit: open-source cheminformatics, 2013. http://www.rdkit.org

29. (a) The Open Babel Package. http://openbabel.org; (b) O'Boyle, N.; Banck, M.; James, C.; Morley, C.; Vandermeersch, T.; Hutchison, G., Open Babel: An open chemical toolbox. Journal of Cheminformatics 2011, 3 (1), 33.

30. O'Boyle, N. M.; Tenderholt, A. L.; Langner, K. M., cclib: A library for packageindependent computational chemistry algorithms. Journal of Computational Chemistry 2008, 29 (5), 839-845.

31. Heller, S.; McNaught, A.; Stein, S.; Tchekhovskoi, D.; Pletnev, I., InChI - the worldwide chemical structure identifier standard. Journal of Cheminformatics 2013, 5 (1), 7.

32. Patchkovskii, S. SYMMETRY. http://www.cobalt.chem.ucalgary.ca/ps/symmetry/

33. Lay, T. H.; Bozzelli, J. W.; Dean, A. M.; Ritter, E. R., Hydrogen Atom Bond Increments for Calculation of Thermodynamic Properties of Hydrocarbon Radical Species. The Journal of Physical Chemistry 1995, 99 (39), 14514-14527.

34. Vandewiele, N. M.; Magoon, G. R.; Van Geem, K. M.; Reyniers, M.-F.; Green, W. H.; Marin, G. B., Experimental and Modeling Study on the Thermal Decomposition of Jet Propellant-10. Energy \& Fuels 2014, 28 (8), 4976-4985.

35. Sharma, S.; Harper, M. R.; Green, W. H., Modeling of 1,3-hexadiene, 2,4-hexadiene and 1,4-hexadiene-doped methane flames: Flame modeling, benzene and styrene formation. Combustion and Flame 2010, 157 (7), 1331-1345.

36. Cavallotti, C.; Polino, D.; Frassoldati, A.; Ranzi, E., Analysis of Some Reaction Pathways Active during Cyclopentadiene Pyrolysis. The Journal of Physical Chemistry A 2012, 116 (13), 3313-3324.

37. CHEMKIN-PRO 15131, Reaction Design: San Diego, 2013.

38. (a) Harper, M. R.; Van Geem, K. M.; Pyl, S. P.; Marin, G. B.; Green, W. H., Comprehensive reaction mechanism for n-butanol pyrolysis and combustion. Combustion and Flame 2011, 158 (1), 16-41; (b) Chen, Q.; Froment, G. F., Thermal-Cracking of Substituted Aromatic-Hydrocarbons .1. Kinetic-Study of the Thermal-Cracking of I-Propylbenzene. Journal of Analytical and Applied Pyrolysis 1991, 21 (1-2), 27-50; (c) Chen, Q.; Froment, G. F., Thermal-Cracking of Substituted Aromatic-Hydrocarbons .2. Kinetic-Study of the ThermalCracking of Normal-Propylbenzene and Ethylbenzene. Journal of Analytical and Applied Pyrolysis 1991, 21 (1-2), 51-77.

39. Murakami, Y.; Saejung, T.; Ohashi, C.; Fujii, N., Investigation of a new pathway forming naphthalene by the recombination reaction of cyclopentadienyl radicals. Chemistry Letters 2003, 32 (12), 1112-1113.

40. Robinson, R. K.; Lindstedt, R. P., On the chemical kinetics of cyclopentadiene oxidation. Combustion and Flame 2011, 158 (4), 666-686.

41. Lindstedt, R. P.; Maurice, L. Q., Detailed kinetic modelling of n-heptane combustion. Combust Sci Technol 1995, 107 (4-6), 317-353. 
42. Zhong, X.; Bozzelli, J. W., Thermochemical and Kinetic Analysis of the H, OH, HO2, O, and O2 Association Reactions with Cyclopentadienyl Radical. The Journal of Physical Chemistry A 1998, 102 (20), 3537-3555. 